\title{
INFINITESIMAL MODULI FOR THE STROMINGER SYSTEM AND KILLING SPINORS IN GENERALIZED GEOMETRY
}

\author{
MARIO GARCIA-FERNANDEZ, ROBERTO RUBIO, AND CARL TIPLER
}

\begin{abstract}
We construct the space of infinitesimal variations for the Strominger system and an obstruction space to integrability, using elliptic operator theory. We initiate the study of the geometry of the moduli space, describing the infinitesimal structure of a natural foliation on this space. The associated leaves are related to generalized geometry and correspond to moduli spaces of solutions of suitable Killing spinor equations on a Courant algebroid. As an application, we propose a unifying framework for metrics with holonomy SU(3) and solutions of the Strominger system.
\end{abstract}

\section{CONTEnTS}

1. Introduction

2. Infinitesimal moduli: abelian case

3. Infinitesimal moduli: general case

4. Anomaly cancellation, flux quantization and generalized geometry

5. Killing spinors in generalized geometry References

\section{INTRODUCTION}

The Strominger system couples a pair of Hermite-Yang-Mills connections with a conformally balanced hermitian metric on a Calabi-Yau threefold $X$, by means of an equation for 4-forms - known as the Bianchi identity. Although originated in string theory [39, 63], its mathematical study was proposed by Yau [70] as a natural generalization of the Calabi problem [11, 69], in relation to moduli spaces of Calabi-Yau threefolds which are not necessarily Kählerian.

Pioneered by Fu, Li and Yau [27, 50], the existence problem for the Strominger system has been an active area of research in mathematics in the last

2010 Mathematics Subject Classification. 58D27, 53D18.

This project has received funding from the European Union's Horizon 2020 research and innovation programme under the Marie Sklodowska-Curie grant agreement No 655162. This work is partially supported by an ESF - Short Visit Grant 5717 within the framework of the ITGP network. MGF is supported by a Marie Sklodowska-Curie grant and was initially supported by ICMAT Severo Ochoa project SEV-2011-0087 and by the École Polytechnique Fédéral de Lausanne. RR is supported by IMPA and was initially supported by QGM through its partnership with the Mathematical Institute of Oxford. CT is partially supported by Agence Nationale de la Recherche - ANR project EMARKS. 
ten years (see $44,20,21,25,26,28,64$ and references therein). There is an important conjecture by Yau [71], which states that any stable holomorphic vector bundle $V$ over a homologically balanced Calabi-Yau threefold $X$ [58] with $c_{2}(V)=c_{2}(X)$ admits a solution of the Strominger system. This conjecture is widely open, the main difficulties being its non-Kähler nature and the lack of understanding of the geometry of the equations.

A problem closely related to Yau's conjecture is the construction of a moduli space of solutions of the Strominger system. This moduli problem remained almost unexplored for a long time, despite its interest in string theory, where it describes the most basic pieces (scalar massless fields) of the four-dimensional theory induced by a heterotic string compactification. Indeed, only a few references that tackle the preliminary question of constructing the tangent space at a given solution can be found in the physics literature [3, 7, 8, 16, 57, 17]. This first step turns out to be rather challenging, and a complete answer has been so far elusive.

The prime motivations for this work are the construction of the moduli space of solutions of the Strominger system and its interrelation with Yau's conjecture. In this paper we make a contribution to the first problem, constructing the space of infinitesimal variations of a solution and an obstruction space to integrability. We initiate the study of the geometry of the moduli space, describing the infinitesimal structure of a natural foliation, whose leaves are intimately related to generalized geometry [37]. By investigating the tangent to a leaf, we give an interpretation of the leaves as moduli spaces of solutions of suitable Killing spinor equations on a Courant algebroid. This last tangent space arises naturally as a quotient of a bigger finite-dimensional vector space by the second de Rham cohomology group of $X$. Our construction provides a unifying framework for metrics with holonomy SU(3) and solutions of the Strominger system, that we expect will have future applications to Yau's conjecture. To explain our results, let us first recall the definition of the equations.

Background. Let $(X, \Omega)$ be a Calabi-Yau threefold, that is, a complex manifold of dimension three endowed with a nowhere vanishing holomorphic section of the canonical bundle $\Omega \in H^{0}\left(X, \Lambda^{3,0} T^{*}\right)$. We do not assume that $X$ is Kählerian. Let $P_{K}$ be a principal bundle over $X$ with compact structure group $K$. The Strominger system is

$$
\begin{aligned}
F^{0,2}=0, & F \wedge \omega^{2}=0, \\
R^{0,2}=0, & R \wedge \omega^{2}=0, \\
d^{*} \omega-i(\bar{\partial}-\partial) \log \|\Omega\|_{\omega} & =0, \\
d d^{c} \omega-\alpha^{\prime}(\operatorname{tr} R \wedge R-\operatorname{tr} F \wedge F) & =0,
\end{aligned}
$$

with unknowns given by a hermitian metric $g$ on $X$ with fundamental form $\omega$, a connection $A$ on $P_{K}$ and a metric connection $\nabla$ on the (smooth) tangent bundle of $X$. Here, $\alpha^{\prime}$ is a positive real constant and $F$ and $R$ denote, respectively, the curvature 2-forms of $A$ and $\nabla$. The notation - tr refers to the Killing form on the Lie algebra of $K$. In this paper we impose that $\nabla$ is unitary with respect to the hermitian structure $(\Omega, \omega)$. 
The Strominger system comprises, essentially, three conditions - the first two well understood in the literature. First, the equation in the third line, often known as the dilatino equation, is strongly reminiscent of the complex MongeAmpère equation on a Kähler manifold (see e.g. [29]). It restricts the holonomy of the Bismut connection

$$
\nabla^{+}=\nabla^{g}-\frac{1}{2} g^{-1}\left(d^{c} \omega\right)
$$

to $\mathrm{SU}(3)$, where $\nabla^{g}$ denotes the Levi-Civita connection of the metric $g$. Furthermore, as observed by $\mathrm{Li}$ and Yau [50], the dilatino equation is equivalent to the condition

$$
d\left(\|\Omega\|_{\omega} \omega^{2}\right)=0,
$$

which implies that $\omega^{\prime}=\|\Omega\|_{\omega}^{1 / 2} \omega$ is the fundamental form of a balanced metric, namely $d^{*} \omega^{\prime}=0$, and hence $g$ is conformally balanced. A classical result of Michelsohn [58] characterizes the existence of balanced metrics on a complex manifold using a condition on the homology - formulated in terms of currents and known as the homologically balanced condition.

Second, the first two lines in (1.1) correspond to the Hermite-Yang-Mills condition for the connections $A$ and $\nabla$ with respect to the conformally balanced metric $g$. There is a well-known theory for Hermite-Yang-Mills connections on a hermitian manifold $(X, g)$ [53, which ranges from existence results to the construction of the moduli space (which turns out to be Kähler when $g$ is conformally balanced). The main result of the theory is Li-Yau's theorem [49], which characterizes the existence of solutions in terms of (slope) stability of the bundle, generalizing the Donaldson-Uhlenbeck-Yau theorem in Kähler geometry [18, 65].

Finally, the most demanding and less understood condition of the system is the Bianchi identity

$$
d d^{c} \omega=\alpha^{\prime}(\operatorname{tr} R \wedge R-\operatorname{tr} F \wedge F)
$$

which is ultimately responsible for the non-Kähler nature of the problem. The non-vanishing of the Pontryagin term $\operatorname{tr} R \wedge R-\operatorname{tr} F \wedge F$ prevents the hermitian form $\omega$ to be closed and hence allows the complex manifold $X$ to be non-Kählerian. This subtle condition, which arises in the quantization of the physical theory, was studied by Freed [24] in the context of index theory for Dirac operators and more recently by Sati-Schreiber-Stasheff from the point of view of differential string structures 62. Despite these important topological insights, we have an almost total lack of understanding of this last equation from an analytical point of view.

Main results. In this work we add to the understanding of the moduli problem for the Strominger system. The first contribution of this work is a complete and direct construction of the vector space of infinitesimal variations of a given solution - the infinitesimal moduli space - using an elliptic complex $S^{*}$. 
Theorem 1.1. The space of infinitesimal deformations of solutions of the Strominger system is given by the first cohomology group $H^{1}\left(S^{*}\right)$ of an elliptic complex of multi-degree differential operators $S^{*}$. This complex admits a natural extension $\tilde{S}^{*}$, and the space of obstructions is defined as $H^{2}\left(\tilde{S}^{*}\right)$.

To clarify the exposition, we first undertake the construction of the complex for a toy model in Section 2. For this, we introduce an abelian version of the equations (1.1) depending on a real parameter. The analysis in the abelian case will show that the combination of the Bianchi identity with the conformally balanced equation (1.2) is well-behaved at the level of symbols.

In Section 3 we construct the elliptic complex of differential operators $S^{*}$ and identify its first cohomology

$$
H^{1}\left(S^{*}\right)
$$

with the infinitesimal moduli of solutions of the Strominger system. Some of the difficulties that arise in the construction of $S^{*}$ come from the symmetries of the system, which turn out to have a Lie groupoid structure due to the compatibility of the connection $\nabla$ with the metric $g$.

In Section 4 we investigate the geometry on the moduli space of solutions of the Strominger system $\mathcal{M}$ derived from the Bianchi identity. This moduli space is endowed with a canonical $H^{3}(X, \mathbb{R})$-valued closed 1 -form

$$
\delta \in \Omega^{1}\left(\mathcal{M}, H^{3}(X, \mathbb{R})\right)
$$

which is constructed via the variation of (1.3). The kernel of $\delta$ defines an integrable distribution on the tangent bundle of $\mathcal{M}$ and hence a foliation on the moduli space. A striking fact about this foliation is that its leaves can be understood by using Hitchin's theory of generalized geometry [37]. The aim of Section 4 is to give a rigorous account of the infinitesimal version of this picture. The construction of a differentiable structure on $\mathcal{M}$ and the local structure of the foliation will be addressed in future work.

Neglecting obstructions to integrability, the tangent to a leaf at a point is defined by an exact sequence

$$
0 \longrightarrow H^{1}\left(\stackrel{\circ}{S^{*}}\right) \longrightarrow H^{1}\left(S^{*}\right) \stackrel{\delta}{\longrightarrow} H^{3}(X, \mathbb{R})
$$

As the notation suggests, $H^{1}\left(\stackrel{\circ}{ }^{*}\right)$ is the cohomology of a complex which, surprisingly, needs generalized geometry for its rigorous definition. In this new framework $H^{1}\left(S^{*}\right)$ has a natural interpretation, as variations of a suitable generalized metric modulo generalized diffeomorphisms. A special feature of $H^{1}\left(\dot{S}^{*}\right)$ is that it cannot be constructed by standard elliptic operator theory, as the space of generalized vector fields cannot be identified with the space of global sections of a vector bundle (similarly as the space of symplectic vector fields on a symplectic manifold). Motivated by this problem, we construct a 
refinement of $H^{1}\left(\stackrel{\circ}{S}^{*}\right)$, which fits into the following exact diagram

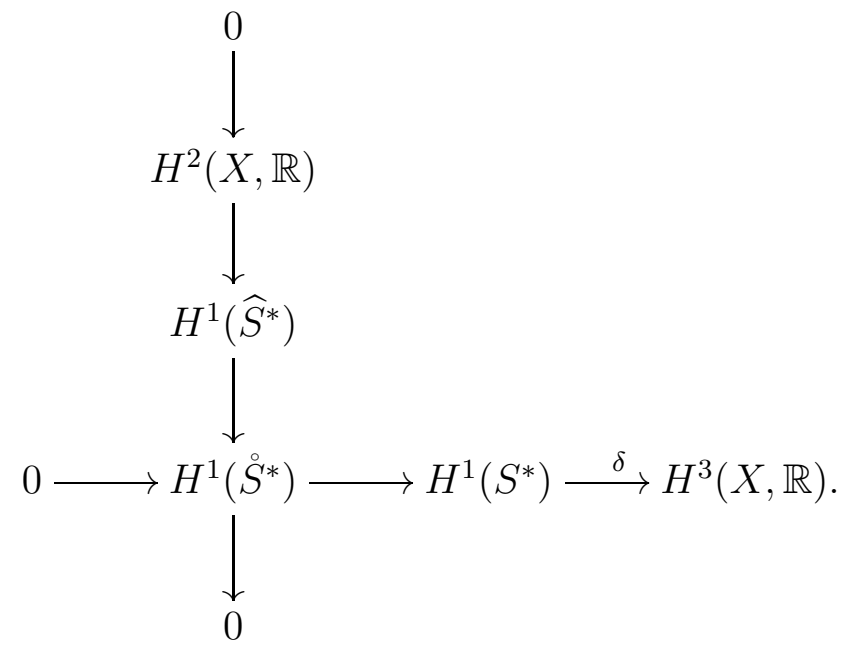

Unlike $H^{1}\left(\stackrel{\circ}{S}^{*}\right)$, the refined vector space $H^{1}\left(\widehat{S}^{*}\right)$ is constructed by considering inner symmetries of a smooth, transitive, Courant algebroid, and is defined as the first cohomology of an elliptic complex of degree 1 differential operators. Motivation for the previous construction comes from two basic principles in the physics of the heterotic string, given by the Green-Schwarz mechanism [34], and the flux quantization condition. We should stress that the space $H^{1}\left(\widehat{S}^{*}\right)$ is the one that comes closer to the physics of the heterotic string.

Section 5 gives a geometric interpretation of the leaves of the foliation in the moduli space, showing the strong connection between the Strominger system and generalized geometry. For this, we define suitable Killing spinor equations

$$
D_{+}^{\phi} \eta=0, \quad \not D_{-}^{\phi} \eta=0,
$$

for an admissible metric on a smooth transitive Courant algebroid and prove the following result.

Theorem 1.2. The Strominger system (1.1) is equivalent to the Killing spinor equations (1.5), on a transitive Courant algebroid obtained from reduction. As a consequence, (1.1) is a natural system of equations in generalized geometry, that is, solutions are exchanged under generalized diffeomorphisms.

This result builds on previous work of the first author in the relation between generalized geometry and heterotic supergravity [30]. Theorem 1.2 gives a precise interpretation of the vector space $H^{1}\left(\stackrel{S}{S}^{*}\right)$, as infinitesimal deformations for solutions to the Killing spinor equations (1.5) modulo infinitesimal symmetries of the Courant algebroid. As a consequence, a leaf of the foliation determined by $\delta$ can be interpreted as a moduli space of solutions of these equations.

The proof of Theorem 1.2 reveals a strong parallelism with the theory of metrics with holonomy $\mathrm{SU}(3)$, once generalized geometry enters into the game. The same equations, formulated instead on an exact Courant algebroid, pin down precisely Riemannian metrics with holonomy SU(3) on a six dimensional manifold. Generalized geometry provides a unifying framework for the theory 
of the Strominger system and the well-established theory of Calabi-Yau metrics, which we expect will have interesting applications in the former. We will explore further this analogy in future work.

In the physics literature, de la Ossa and Svanes [17] and Anderson, Gray and Sharpe [3] have recently proposed a close approximation to the infinitesimal moduli for the Strominger system in terms of the Dolbeault cohomology of a holomorphic double extension-based on previous ideas by Melnikov and Sharpe [57]. In a sequel to the present paper we will show that their proposal admits a natural interpretation in generalized geometry, and relates in a certain way to the infinitesimal moduli of the Strominger system. After Section 5 was completed, we were informed by A. Coimbra that an alternative formulation of the Strominger system using generalized geometry was provided recently in the physics literature [15]. Our approach has the benefit of making evident that the Strominger system is invariant under generalized diffeomorphisms.

Acknowledgments: We thank Luis Álvarez-Cónsul, Bjorn Andreas, Vestislav Apostolov, Henrique Bursztyn, Ryushi Goto, Marco Gualtieri, Nigel Hitchin, Laurent Meersseman, Xenia de la Ossa, Dan Popovici, Brent Pym and Eirik Svanes for useful discussions. Part of this work was undertaken while CT was visiting IMPA, UFRJ, CRM, during visits of MGF and CT to CIRGET, and of RR to EPFL and ICMAT. We would like to thank these very welcoming institutions for providing a nice and stimulating working environment.

\section{INFINITESIMAL MODULI: ABELIAN CASE}

The aim of this section is to study a toy model for the construction of the infinitesimal moduli space of the Strominger system, which avoids the difficulties arising from the treatment of the unitary connection on the tangent bundle and non-abelian groups. In particular, we will consider deformations of a Calabi-Yau structure on a compact, six dimensional, smooth manifold $M$, endowed with a holomorphic line bundle. We do not require our complex manifolds to be Kählerian.

2.1. The abelian equations. Let $M$ be a compact, oriented, six dimensional smooth manifold. Let $L$ be a hermitian line bundle over $M$. We fix a non-zero real constant $c$. We denote by $T$ the smooth tangent bundle of $M$ and its complexification by $T_{\mathbb{C}}$. Consider triples $(\Omega, \theta, \omega)$ where $\Omega$ is a complex 3 -form such that

$$
T^{0,1}:=\left\{V \in T_{\mathbb{C}} \mid \iota_{V} \Omega=0\right\}
$$

determines an almost complex structure $J_{\Omega}$ on $M, \theta$ is a unitary connection

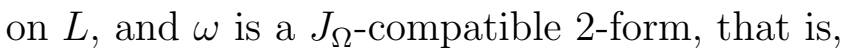

$$
\omega\left(J_{\Omega} \cdot J_{\Omega} \cdot\right)=\omega \text { and } \omega\left(\cdot, J_{\Omega} \cdot\right)>0
$$


is a Riemannian metric on $M$. We aim to construct a space of infinitesimal deformations for solutions of the equations

$$
\begin{aligned}
& d \Omega=0, \quad d\left(\|\Omega\|_{\omega} \omega^{2}\right)=0, \\
& F_{\theta}^{0,2}=0, \quad F_{\theta} \wedge \omega^{2}=\lambda \omega^{3}, \\
& d d^{c} \omega-c\left(F_{\theta} \wedge F_{\theta}\right)=0,
\end{aligned}
$$

where $\lambda \in i \mathbb{R}$ is a constant that depends on the unitary structure determined by $(\Omega, \omega)$ and the first Chern class of $L$, as follows from the identity

$$
\operatorname{deg}(L):=c_{1}(L) \cdot\left[\|\Omega\|_{\omega} \omega^{2}\right]=\frac{i \lambda}{2 \pi} \int_{M}\|\Omega\|_{\omega} \omega^{3} .
$$

Our convention for the point-wise norm of a $(3,0)$-form $\varphi$ with respect to $\omega$ is

$$
\|\varphi\|_{\omega}^{2} \omega^{3}=6 i \varphi \wedge \bar{\varphi}
$$

Recall that the integrability of $J_{\Omega}$ is equivalent to the condition

$$
d \Omega=0
$$

and hence, by the first two equations in (2.3), any solution determines a CalabiYau threefold structure on $M$ endowed with a holomorphic line bundle.

When $c_{1}(L)=0$ and $c=-1$ the system (2.3) corresponds to the field equations of abelian heterotic supergravity considered in [55] (note that in our notation $F_{\theta}$ is a purely imaginary 2 -form). The reason why we do not work directly with this case, which is the situation that comes closer to the Strominger system, is the following observation, that shall be compared with [12, p. 55].

Proposition 2.1. Let $(\Omega, \theta, \omega)$ be a solution of (2.3). If $c_{1}(L)=0$ and $(M, \Omega)$ is a $\partial \bar{\partial}$-manifold, then $\theta$ is flat and $\omega$ has holonomy $\mathrm{SU}(3)$ (in particular, $\omega$ is Kähler Ricci-flat).

Proof. As $c_{1}(L)=0, F_{\theta}$ is exact. By the $\partial \bar{\partial}$-lemma, $F_{\theta}=\partial \bar{\partial} f$ for some smooth function $f$ on $X$. After conformal re-scaling of the hermitian metric on $L$, we obtain a flat Chern connection on the holomorphic line bundle $\left(L, \bar{\partial}_{\theta}\right)$. Then, since $\theta$ is the Chern connection of a hermitian-Einstein metric on $L, \theta$ has to be flat by uniqueness, and hence it follows that $\omega$ is strong Kähler with torsion. By the conformally balanced condition, the Bismut connection of $\omega$ has holonomy SU(3) (see [63, Section II]). Applying now [41, Corollary 4.7], $\omega$ is Kähler and the result follows.

In this work we are mainly interested in non-Kähler solutions of (2.3), and therefore we will assume that $c_{1}(L) \neq 0$. Non-Kähler solutions of (2.3) can be obtained using the perturbative method in [4], from holomorphic line bundles $L$ over a projective Calabi-Yau threefold $X$ with non-torsion $c_{1}(L) \in H^{2}(X, \mathbb{Z})$ satisfying

$$
c_{1}(L)^{2}=0 .
$$

Remark 2.1. It is perhaps more natural to consider the Hermite-Yang-Mills equations in $(2.3)$ with respect to the conformally balanced metric $\|\Omega\|_{\omega}^{\frac{1}{2}} \omega$. The 
linearization of these alternative abelian equations is, however, more involved and does not add to the understanding of the Strominger system.

2.2. Notation, parameter space and symmetries. Let $\Omega$ be a complex 3 -form on $M$ such that (2.1) determines an almost complex structure $J$ on $M$ with anti-holomorphic tangent bundle $T^{0,1}$. Denote by $\Omega^{k}$ (resp. $\Omega_{\mathbb{C}}^{k}$ ) the space of real (resp. complex) smooth $k$-forms on $M$. We denote by $\Omega^{p, q}$ the space of $(p, q)$-forms on $(M, J)$ and by $\Omega^{p, q}(W)$ the space of $(p, q)$-forms taking values in a vector bundle $W$. Given a $q$-form $\sigma$ on $M$ (that may take values in a vector bundle) and $\gamma \in \Omega^{p}\left(T_{\mathbb{C}}\right)$, we define a $(q+p-1)$-form $\sigma^{\gamma}$ by

$$
\sigma^{\gamma}=(\sigma(\gamma, \cdot))^{s k w}
$$

where $s k w$ denotes the skew-symmetric part of the tensor $\sigma(\gamma, \cdot)$ satisfying :

$\forall\left(V_{j}\right) \in\left(T_{\mathbb{C}}\right)^{q+p-1}, \sigma(\gamma, \cdot)\left(V_{1}, \ldots, V_{q+p-1}\right)=\sigma\left(\gamma\left(V_{1}, \cdots, V_{p}\right), V_{p+1}, \ldots, V_{q+p-1}\right)$.

As $\Omega$ is nowhere vanishing, it induces an isomorphism on forms

$$
\begin{aligned}
T_{0}: \Omega^{0, j}\left(T^{1,0}\right) & \rightarrow \Omega^{2, j} \\
\gamma & \mapsto \Omega^{\gamma} .
\end{aligned}
$$

We will also denote by $T_{0}$ the induced isomorphism in cohomology [59].

To study the infinitesimal moduli of (2.3) we define the following parameter space. Let $\mathcal{A}$ be the space of unitary connections on $L$ and $\Omega_{0}^{3} \subset \Omega_{\mathbb{C}}^{3}$ the non-linear subspace of complex 3 -forms such that (2.1) determines an almost complex structure on $M$. We set

$$
\mathcal{P}:=\left\{(\Omega, \theta, \omega) \mid \omega \text { is } J_{\Omega}-\text { compatible }\right\} \subset \Omega_{0}^{3} \times \mathcal{A} \times \Omega^{2},
$$

where the compatibility condition is as in (2.2).

Let Diff $_{0}$ be the identity component of the group of diffeomorphisms of $M$. Consider the group $\widetilde{\mathcal{G}}$ of automorphisms of $L$ that preserve the unitary bundle structure and cover an element in Diff ${ }_{0}$. This group preserves $\mathcal{P}$, exchanging solutions of (2.3). Denote by $\mathcal{G}$ the gauge group of the hermitian bundle $L$. Then we have an exact sequence [1]

$$
1 \rightarrow \mathcal{G} \longrightarrow \widetilde{\mathcal{G}} \stackrel{p}{\longrightarrow} \operatorname{Diff}_{0} \rightarrow 1 \text {. }
$$

Given a connection $\theta$ on $L$, we have a lift $\Omega^{0}(T) \rightarrow \operatorname{Lie}(\widetilde{\mathcal{G}})$ and at the level of vector spaces the corresponding Lie algebra sequence splits

$$
0 \rightarrow \operatorname{Lie}(\mathcal{G}) \longrightarrow \operatorname{Lie}(\widetilde{\mathcal{G}}) \stackrel{p}{\longrightarrow} \Omega^{0}(T) \rightarrow 0 .
$$

2.3. Linearization and ellipticity. In the sequel, we fix a solution $(\Omega, \theta, \omega)$ of (2.3). The integrable almost complex structure determined by $\Omega$ will be denoted by $J$ and the curvature of $\theta$ will be denoted by $F$. The complex encoding infinitesimal deformations of (2.3) is built from an elliptic complex parameterizing infinitesimal deformations of the complex structure that preserve the Calabi-Yau condition, that is, with trivial canonical bundle, 33]

$$
0 \rightarrow \Omega^{0}\left(T^{1,0}\right) \stackrel{\mathcal{L} \cdot \Omega}{\longrightarrow} \Omega^{3,0} \oplus \Omega^{2,1} \stackrel{d}{\longrightarrow} \Omega^{3,1} \oplus \Omega^{2,2} .
$$


Here, the first non-trivial arrow is defined by the infinitesimal action of Diff ${ }_{0}$, given by the Lie derivative of $\Omega$ and we use the following characterization of the tangent space of $\Omega_{0}^{3}$ at $\Omega$ :

$$
T_{\Omega} \Omega_{0}^{3}=\Omega^{3,0} \oplus \Omega^{2,1} .
$$

The variations in $\Omega^{3,0}$ are just rescaling of the holomorphic 3-form, while elements in $\Omega^{2,1}$ correspond via $T_{0}$ to deformations of the complex structure. Given $\dot{\Omega} \in \Omega^{3,0} \oplus \Omega^{2,1}$, we will denote by $\dot{J}$ the associated variation of almost complex structure given by

$$
\dot{J}^{1,0}=2 i T_{0}^{-1}\left(\dot{\Omega}^{2,1}\right),
$$

with $\dot{J}^{1,0}=\frac{1}{2}(\dot{J}-i J \dot{J})$.

Let $T_{0} \mathcal{P}$ be the tangent space of $\mathcal{P}$ at the initial solution:

$$
T_{0} \mathcal{P}=\left\{(\dot{\Omega}, \dot{\theta}, \dot{\omega}) \in \Omega^{3,0} \oplus \Omega^{2,1} \oplus \Omega^{1}(i \mathbb{R}) \oplus \Omega^{2} \mid J \dot{\omega}-\dot{\omega}=-\omega^{j J}\right\} .
$$

Note that the equations that define $T_{0} \mathcal{P}$ can be equivalently written as

$$
2 i \dot{\omega}^{0,2}=\omega^{j^{1,0}}
$$

and therefore there is a canonical isomorphism

$$
T_{0} \mathcal{P} \cong A^{1}:=\Omega^{3,0} \oplus \Omega^{2,1} \oplus \Omega^{1}(i \mathbb{R}) \oplus \Omega_{\mathbb{R}}^{1,1},
$$

where $\Omega_{\mathbb{R}}^{1,1} \subset \Omega^{1,1}$ denotes the space of real $(1,1)$-forms on $(M, \Omega)$, given explicitly by

$$
\dot{\omega}=\dot{\omega}^{1,1}+\frac{1}{2} \omega^{\dot{J} J} .
$$

Consider the linearization of the equations (2.3)

$$
\mathbf{L}: T_{0} \mathcal{P} \rightarrow A^{2}:=\Omega^{3,1} \oplus \Omega^{2,2} \oplus \Omega^{0,2} \oplus \Omega^{4} \oplus \Omega^{5} \oplus \Omega^{6}(i \mathbb{R}) .
$$

Using the vector space splitting (2.10) given by the fixed connection $\theta$, the infinitesimal action

$$
\mathbf{P}: \operatorname{Lie}(\widetilde{\mathcal{G}}) \rightarrow T_{0} \mathcal{P}
$$

reads explicitly

$$
\begin{array}{rlc}
\mathbf{P}: \quad A^{0}:=\Omega^{0}(T) \times \Omega^{0}(i \mathbb{R}) & \rightarrow & T_{0} \mathcal{P} \\
(V, r) & \mapsto\left(d \iota_{V^{1,0}} \Omega, \iota_{V} F+d r, \mathcal{L}_{V} \omega\right)
\end{array}
$$

with $V^{1,0}=\frac{1}{2}(V-i J V)$. We construct a complex of differential operators

$$
A^{0} \stackrel{\mathbf{P}}{\longrightarrow} A^{1} \stackrel{\mathbf{L}}{\longrightarrow} A^{2},
$$

combining the operators $\mathbf{P}$ and $\mathbf{L}$ with the isomorphism (2.14). Our aim is to prove that this complex is elliptic. Note that an arbitrary unitary connection on $L$ is of the form $\theta+\dot{\theta}$ where $\dot{\theta} \in \Omega^{1}(i \mathbb{R})$, with corresponding curvature $F+d \dot{\theta}$, and also that $\lambda=\lambda(\Omega, \omega)$ is a function of the hermitian structure given by (2.4). Using this, we obtain the following expression for the differential $\mathbf{L}$, regarded as an operator with domain $T_{0} \mathcal{P}$. 
Lemma 2.1. The differential $\mathbf{L}=\oplus_{i=1}^{5} \mathbf{L}_{i}$ is given by

$$
\begin{array}{lc}
\mathbf{L}_{1}(\dot{\Omega}, \dot{\theta}, \dot{\omega})= & d \dot{\Omega}, \\
\mathbf{L}_{2}(\dot{\Omega}, \dot{\theta}, \dot{\omega})= & \bar{\partial} \dot{\theta}^{0,1}+\frac{i}{2}\left(F^{j}\right)^{0,2}, \\
\mathbf{L}_{3}(\dot{\Omega}, \dot{\theta}, \dot{\omega})= & d\left(J d \dot{\omega}-J(d \omega)^{\dot{j} J}-2 c(\dot{\theta} \wedge F)\right), \\
\mathbf{L}_{4}(\dot{\Omega}, \dot{\theta}, \dot{\omega})= & d\left(2|| \Omega \|_{\omega} \dot{\omega} \wedge \omega+\delta\left(\|\Omega\|_{\omega}\right) \omega^{2}\right), \\
\mathbf{L}_{5}(\dot{\Omega}, \dot{\theta}, \dot{\omega})= & d \dot{\theta} \wedge \omega^{2}+(2 F-3 \lambda \omega) \wedge \dot{\omega} \wedge \omega-\dot{\lambda} \omega^{3} .
\end{array}
$$

where $\dot{J}$ and $\dot{\lambda}$ are, respectively, the infinitesimal variations of almost-complex structure and constant $\lambda$ defined by $\dot{\Omega}$ and $\dot{\omega}$, and

$$
\delta\left(\|\Omega\|_{\omega}\right)=\|\Omega\|_{\omega}^{-1} \operatorname{Re}(\dot{\Omega}, \Omega)_{\omega}-\frac{1}{2}\|\Omega\|_{\omega} \Lambda_{\omega} \dot{\omega} .
$$

Proof. The calculation of $\mathbf{L}_{1}, \mathbf{L}_{4}$ and $\mathbf{L}_{5}$ is straightforward and for the calculation of $\mathbf{L}_{2}$ see e.g. [31]. Formula (2.19) follows from (2.5). To compute $\mathbf{L}_{3}$, note that $d^{c}=J d J^{-1}$, where the action of $J$ on forms $b \in \Omega^{p}$ is

$$
J b=b\left(J^{-1} \cdot, \ldots, J^{-1} \cdot\right)=(-1)^{p} b(J \cdot, \ldots, J \cdot) .
$$

Then, using the compatibility between $\omega$ and $J_{\Omega}$ in (2.3) we have $d_{J_{\Omega}}^{c} \omega=J_{\Omega} d \omega$ and therefore

Lastly,

$$
\frac{d}{d t}_{\mid t=0}\left(J_{t} d J_{t}^{-1} \omega_{t}\right)=\frac{d}{d t}_{\mid t=0}\left(J_{t}(d \omega)\right)+J d \dot{\omega}
$$

$$
\begin{aligned}
{\left.\frac{d}{d t}\right|_{t=0}\left(J_{t} d \omega\right)} & -d \omega(\dot{J} \cdot, J \cdot, J \cdot)-d \omega(J \cdot, \dot{J} \cdot, J \cdot)-d \omega(J \cdot, J \cdot, \dot{J} \cdot) \\
& =d \omega\left(\dot{J} J^{2} \cdot, J \cdot, J \cdot\right)+d \omega\left(J \cdot, \dot{J} J^{2} \cdot, J \cdot\right)+d \omega\left(J \cdot, J \cdot, \dot{J} J^{2} \cdot\right) \\
& =-J(d \omega(\dot{J} J \cdot, \cdot, \cdot)+d \omega(\cdot, \dot{J} J \cdot, \cdot)+d \omega(\cdot, \cdot, \dot{J} J \cdot)) \\
& =-J(d \omega)^{\dot{J} J} .
\end{aligned}
$$

We note that the differential operator $\mathbf{L}$ is of first order in the components $\mathbf{L}_{1}, \mathbf{L}_{2}, \mathbf{L}_{4}$ and $\mathbf{L}_{5}$, but $\mathbf{L}_{3}$ has order two. We shall use the generalized notion of ellipticity provided by Douglis and Nirenberg [19]. For the general theory of linear multi-degree elliptic differential operators we refer to [51, 52]. Here we recall the basic definition. Let $E$ and $F$ be smooth real vector bundles over the compact manifold $M$ with a direct sum decomposition

$$
E=\bigoplus_{j=1}^{m} E_{j}, \quad F=\bigoplus_{i=1}^{l} F_{i},
$$

and $\mathbf{L}: \Omega^{0}(E) \rightarrow \Omega^{0}(F)$ a linear differential operator with corresponding decomposition $\mathbf{L}=\oplus_{i, j} \mathbf{L}_{i j}$. 
Definition 2.1. Two tuples, $\mathbf{t}=\left(t_{1}, \ldots, t_{m}\right)$ and $\mathbf{s}=\left(s_{1}, \ldots, s_{l}\right)$ of nonnegative integers form a system of orders for $\mathbf{L}$ if for each $1 \leq j \leq m, 1 \leq i \leq l$ we have order $\mathbf{L}_{i j} \leq t_{j}-s_{i}$ (if $t_{j}-s_{i}<0$ then $\left.\mathbf{L}_{i j}=0\right)$. The $(\mathbf{t}, \mathbf{s})$-principal part of $\mathbf{L}$ is obtained by replacing each $\mathbf{L}_{i j}$ by its terms which are exactly of order $t_{j}-s_{i}$, and the $(\mathbf{t}, \mathbf{s})$-principal symbol of $\mathbf{L}$ is obtained by replacing each $\mathbf{L}_{i j}$ with its $t_{j}-s_{i}$ principal symbol.

We apply now this definition to our setup.

Lemma 2.2. The leading symbol of $\mathbf{P}$ is given by the formula

$$
\sigma_{\mathbf{P}}(v)(V, r)=\left(v \wedge \iota_{V} \Omega, v r, v \wedge \iota_{V} \omega\right),
$$

where $v \in T^{*} \backslash M$. The tuples $\mathbf{t}=(2,2,2)$ and $\mathbf{s}=(1,1,0,1,1)$ form a system of orders for $\mathbf{L}$ and the associated leading symbol is

$$
\sigma_{\mathbf{L}}(v)(\dot{\Omega}, \dot{\theta}, \dot{\omega})=\left(\sigma_{\mathbf{L}_{1}}(v), \sigma_{\mathbf{L}_{2}}(v), \sigma_{\mathbf{L}_{3}}(v), \sigma_{\mathbf{L}_{4}}(v), \sigma_{\mathbf{L}_{5}}(v)\right),
$$

with

$$
\begin{array}{lc}
\sigma_{\mathbf{L}_{1}}(v)(\dot{\Omega}, \dot{\theta}, \dot{\omega})= & v \wedge \dot{\Omega}, \\
\sigma_{\mathbf{L}_{2}}(v)(\dot{\Omega}, \dot{\theta}, \dot{\omega})= & (v \wedge \dot{\theta})^{(0,2),} \\
\sigma_{\mathbf{L}_{3}}(v)(\dot{\Omega}, \dot{\theta}, \dot{\omega})= & v \wedge J(v \wedge \dot{\omega}), \\
\sigma_{\mathbf{L}_{4}}(v)(\dot{\Omega}, \dot{\theta}, \dot{\omega})= & v \wedge\left(2\|\Omega\|_{\omega} \dot{\omega} \wedge \omega+\delta\left(\|\Omega\|_{\omega}\right) \omega^{2}\right), \\
\sigma_{\mathbf{L}_{5}}(v)(\dot{\Omega}, \dot{\theta}, \dot{\omega})= & v \wedge \dot{\theta} \wedge \omega^{2} .
\end{array}
$$

Proof. To apply Definition 2.1 we use the direct sum decomposition (2.14), setting $\Omega^{0}\left(E_{1}\right)=\Omega^{3,0} \oplus \Omega^{2,1}, \Omega^{0}\left(E_{2}\right)=\Omega^{1}(i \mathbb{R})$ and $\Omega^{0}\left(E_{3}\right)=\Omega_{\mathbb{R}}^{1,1}$. The proof is a routine check and is left to the reader.

A linear multi-degree complex of differential operators is elliptic if the induced sequence of symbols is exact, as in the standard case. The usual Fredholm properties of elliptic complexes hold, and therefore given any elliptic complex we have an associated finite dimensional cohomology.

Proposition 2.2. The sequence (2.17) is an elliptic complex. The space of infinitesimal deformations of the system (2.3) is defined as the finite-dimensional vector space

$$
H^{1}\left(A^{*}\right)=\frac{\operatorname{Ker} \mathbf{L}}{\operatorname{Im} \mathbf{P}}
$$

Proof. By $\widetilde{\mathcal{G}}$-invariance of $(2.3), \mathbf{L} \circ \mathbf{P}=0$. We prove next that the associated sequence of symbols is exact. Assume that $\sigma_{\mathbf{L}}(v)(\dot{\Omega}, \dot{\theta}, \dot{\omega})=0$ for $v \in T^{*} \backslash M$. From the equations $\sigma_{\mathbf{L}_{j}}(v)=0, j=2,5$, we deduce that there is a purely imaginary constant $r$ such that $\dot{\theta}=v r$. Using the ellipticity of the complex (2.11) and the isomorphism (2.7) there exists a unique $V \in T$ such that

$$
\dot{\Omega}=v \wedge \iota_{V} \Omega \text {. }
$$


In terms of $\dot{J}$, this translates to

$$
\dot{J}=J v \otimes V+v \otimes J V .
$$

It remains to show that $\dot{\omega}=v \wedge \iota_{V} \omega$. From $\sigma_{\mathbf{L}_{4}}(v)=0$ we deduce

$$
(J v) \wedge v \wedge\left(2|| \Omega \|_{\omega} \dot{\omega} \wedge \omega+\delta\left(\|\Omega\|_{\omega}\right) \omega^{2}\right)=0 .
$$

Using now $J\left(\sigma_{\mathbf{L}_{3}}(v)\right)=0$, we obtain

$$
\delta\left(\|\Omega\|_{\omega}\right)(J v) \wedge v \wedge \omega^{2}=0,
$$

and from this,

$$
\delta\left(\|\Omega\|_{\omega}\right)=0
$$

Using now (2.5) and (2.19)

$$
\begin{aligned}
\|\Omega\|_{\omega}^{2} \dot{\omega} \wedge \omega^{2} & =2 i(\dot{\Omega} \wedge \bar{\Omega}+\Omega \wedge \overline{\dot{\Omega}}) \\
& =2 i v \wedge i_{V}(\Omega \wedge \bar{\Omega}) \\
& =\|\Omega\|_{\omega}^{2} v \wedge\left(i_{V} \omega\right) \wedge \omega^{2} .
\end{aligned}
$$

Define $\tau=\dot{\omega}-v \wedge\left(i_{V} \omega\right)$ and notice from (2.13) and (2.23) that $\tau$ is a real (1,1)-form. Furthermore, from (2.26) and (2.25), combined with the vanishing of $\sigma_{\mathbf{L}_{3}}(v)$ and $\sigma_{\mathbf{L}_{4}}(v)$, we deduce

$$
\begin{aligned}
\tau \wedge \omega^{2} & =0, \\
\tau \wedge v \wedge \omega & =0, \\
\tau \wedge v \wedge J v & =0 .
\end{aligned}
$$

From the last equation

$$
\tau=v \wedge a+J v \wedge b
$$

for suitable 1-forms $a$ and $b$. Complete the family $\{v, J v\}$ into a basis of $T^{*}$ with forms $\{X, J X, Y, J Y\}$ such that $\omega$ is written

$$
\omega=l v \wedge J v+X \wedge J X+Y \wedge J Y
$$

for some real constant $l$. Now, from the second equation in (2.27) we obtain that $b$ is proportional to $v$ and therefore

$$
\tau=v \wedge\left(t_{1} J v+t_{2} X+t_{3} J X+t_{4} Y+t_{5} J Y\right)
$$

for suitable real constants $t_{1}, \ldots, t_{5} \in \mathbb{R}$. We note that a basis of the space of real $(1,1)$-forms is

$$
\begin{aligned}
\Lambda_{\mathbb{R}}^{1,1}= & \langle v \wedge J v, X \wedge J X, Y \wedge J Y, X \wedge v+J X \wedge J v, \\
& J X \wedge v+J v \wedge X, Y \wedge v+J Y \wedge J v, J Y \wedge v+J v \wedge Y, \\
& X \wedge Y+J X \wedge J Y, J X \wedge Y+J Y \wedge X\rangle
\end{aligned}
$$

and therefore necessarily

$$
\tau=t_{1} v \wedge J v .
$$

Finally, from the first equation in (2.27) we obtain $t_{1}=0$, which implies that $\dot{\omega}=v \wedge\left(i_{V} \omega\right)$ as claimed. 
2.4. Extension of the complex. We extend the complex (2.17) in order to define a space of obstructions to integrability of infinitesimal deformations of the abelian equations (2.3).

We first define a complex parameterizing joint deformations of a Calabi-Yau structure on $M$ endowed with a holomorphic line bundle. For this, we combine an elliptic complex defined by Goto [33] with previous work of Huang [38]. Goto's complex is an extension of the complex (2.11) given by

$$
0 \rightarrow \Omega^{2,0} \stackrel{d}{\longrightarrow} \Omega^{3,0} \oplus \Omega^{2,1} \stackrel{d}{\longrightarrow} \Omega^{3,1} \oplus \Omega^{2,2} \stackrel{d}{\longrightarrow} \Omega^{3,2} \oplus \Omega^{2,3} \stackrel{d}{\longrightarrow} \Omega^{3,3} \longrightarrow 0,
$$

where we use the identification $\Omega^{0}\left(T^{1,0}\right) \cong \Omega^{2,0}$ provided by the isomorphism (2.7). Following [38] (cf. [31]) we define an elliptic complex

$$
0 \rightarrow C^{0} \stackrel{\bar{\partial}_{0}}{\longrightarrow} C^{1} \stackrel{\bar{\partial}_{0}}{\longrightarrow} C^{2} \stackrel{\bar{\partial}_{0}}{\longrightarrow} C^{3} \stackrel{\bar{\partial}_{0}}{\longrightarrow} C^{4} \longrightarrow 0
$$

where

$$
C^{j}:=\Omega^{3, j-1} \oplus \Omega^{2, j} \oplus \Omega^{0, j}, \quad j \geq 0,
$$

(by convention $\Omega^{3,-1}=0$ ) with differential given by

$$
\bar{\partial}_{0}(\alpha, \beta)=\left(d \alpha, \bar{\partial} \beta-F^{T_{0}^{-1}\left(\alpha^{2, j}\right)}\right) .
$$

To include deformations of the metric, we build on the complex for the Hermite-Yang-Mills equations defined by Kim [44] (see also [45, p. 246]). Consider the following commutative diagram

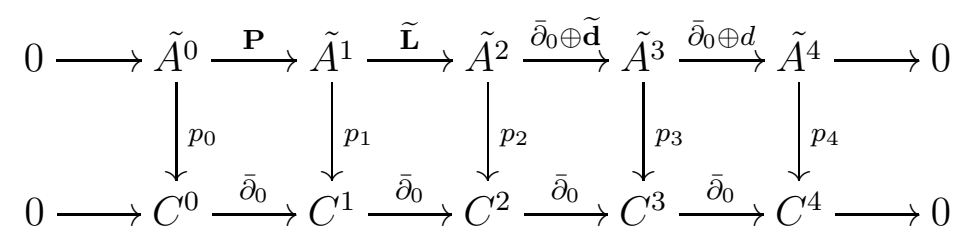

with

$$
\begin{array}{rlrl}
\tilde{A}^{0}:=A^{0}, & p_{0}(V, r)=\left(i_{V} \Omega, r\right), \\
\tilde{A}^{1}:=A^{1}, & p_{1}(\dot{\Omega}, \dot{\theta}, \dot{\omega})=\left(\dot{\Omega}, \dot{\theta}^{0,1}\right), \\
\tilde{A}^{2}:=C^{2} \oplus \Omega_{\mathbb{R}}^{2,2} \oplus \Omega^{5} \oplus \Omega^{6}(i \mathbb{R}), & & p_{2}(s, \gamma, \delta, \epsilon)=s, \\
\tilde{A}^{j}:=C^{j} \oplus \Omega^{j+2} \oplus \Omega^{j+3}, & & p_{j}(s, \tau, \sigma)=s, \quad j=3,4,
\end{array}
$$

where $s$ denotes an element in $C^{j}$ for $j \geq 2$. It remains to define the maps $\widetilde{\mathbf{L}}$ and $\widetilde{\mathbf{d}}$ in (2.31), given by suitable modifications of the operator $\mathbf{L}$ in (2.17) (see Lemma 2.1) and the exterior differential. Firstly, we define

$$
\widetilde{\mathbf{L}}=\mathbf{L}_{1} \oplus \mathbf{L}_{2} \oplus \mathbf{L}_{3}^{2,2} \oplus \mathbf{L}_{4} \oplus \mathbf{L}_{5},
$$

where $\mathbf{L}_{3}^{2,2}$ denotes the $(2,2)$ part of $\mathbf{L}_{3}$ in (2.18). We note that $d \circ \mathbf{L}_{3}=0$, but $d \circ \mathbf{L}_{3}^{2,2}$ does not vanish in general. In fact, we have the following formula.

Lemma 2.3. The $(1,3)$ part of $\mathbf{L}$ is given by

$$
\mathbf{L}_{3}^{1,3}=-2 i \partial\left(\omega^{T_{0}^{-1}\left(\mathbf{L}_{1}^{2,2}\right)}\right)-2 i(\partial \omega)^{T_{0}^{-1}\left(\mathbf{L}_{1}^{2,2}\right)}-2 c \mathbf{L}_{2} \wedge F .
$$


This motivates the introduction of the map

$$
\widetilde{\mathbf{d}}: \tilde{A}^{2} \rightarrow \Omega^{5} \oplus \Omega^{6},
$$

defined by

$$
\widetilde{\mathbf{d}}(\alpha, \beta, \gamma, \delta, \epsilon)=\left(d\left(\gamma-2 \operatorname{Re}\left(2 i(\partial \omega)^{T_{0}^{-1}\left(\alpha^{2,2}\right)}+2 c \beta \wedge F\right)\right), d \delta\right)
$$

We are now ready to prove the main result of this section.

Proposition 2.3. The sequence $\tilde{A}^{*}$ defines an elliptic complex of differential operators, whose first cohomology $H^{1}\left(\tilde{A}^{*}\right)$ equals the cohomology $H^{1}\left(A^{*}\right)$ of (2.17). The space of obstructions for the system (2.3) is defined as $H^{2}\left(\tilde{A}^{*}\right)$.

Remark 2.2. The complex $\tilde{A}^{*}$ has a slightly different flavour from the one of Kim [44] (see also [45]), due to the conformally balanced equation. In Kim's complex, the linearization of the hermitian-Yang-Mills equation is extended by zero, while in (2.31) we need to introduce an exterior differential to extend the linearization of the conformally balanced equation as part of an elliptic complex.

We start with the proof of (2.32). We need the following.

Lemma 2.4. Let $\gamma \in \Omega^{d}\left(T^{1,0}\right)$ and $\alpha \in \Omega^{p, q}$. Then

$$
\bar{\partial}\left(\alpha^{\gamma}\right)=\alpha^{\bar{\partial} \gamma}-(-1)^{d}(\bar{\partial} \alpha)^{\gamma} \text {. }
$$

Proof. Write locally $\gamma=\sum_{k} \gamma_{k} \frac{\partial}{\partial z_{k}}$ so that

$$
\alpha^{\gamma}=\sum_{k} \gamma_{k} \wedge \iota \frac{\partial}{\partial z_{k}} \alpha
$$

Then

$$
\bar{\partial} \alpha^{\gamma}=\sum_{k}\left(\bar{\partial} \gamma_{k}\right) \wedge \iota_{\frac{\partial}{\partial z_{k}}} \alpha+(-1)^{d} \sum_{k} \gamma_{k} \wedge \bar{\partial}\left(\iota_{\frac{\partial}{\partial z_{k}}} \alpha\right)
$$

The formula

$$
\bar{\partial}\left(\iota \frac{\partial}{\partial z_{k}} \alpha\right)=-\iota \frac{\partial}{\partial z_{k}} \bar{\partial} \alpha
$$

follows from $\iota_{\frac{\partial}{\partial z_{k}}} d \bar{z}_{j}=0$ and the expression of $\alpha$ in local coordinates.

Proof of Lemma 2.3. By (2.18) and (2.13)

$$
\begin{aligned}
\left(\mathbf{L}_{3}(\dot{\Omega}, \dot{\theta}, \dot{\omega})\right)^{(3,1)+(1,3)}= & -\partial \bar{\partial}\left(\omega^{j}\right)-\left(d J(d \omega)^{\dot{J} J}\right)^{(3,1)+(1,3)} \\
& -2 c\left(\partial \dot{\theta}^{1,0}+\bar{\partial} \dot{\theta}^{0,1}\right) \wedge F
\end{aligned}
$$

and

$$
\left(d J(d \omega)^{j J}\right)^{1,3}=\bar{\partial}\left((\partial \omega)^{\dot{j}^{1,0}}\right)-\partial\left((\bar{\partial} \omega)^{\dot{j}^{1,0}}\right) .
$$

From formula (2.33), we obtain

$$
\begin{aligned}
\left(\mathbf{L}_{3}(\dot{\Omega}, \dot{\theta}, \dot{\omega})\right)^{(1,3)}= & -\partial \bar{\partial}\left(\omega^{\dot{j}^{1,0}}\right)-\bar{\partial}\left((\partial \omega)^{\dot{j}^{1,0}}\right)+\partial\left((\bar{\partial} \omega)^{\dot{j}^{1,0}}\right) \\
& -2 c\left(\bar{\partial} \dot{\theta}^{0,1}\right) \wedge F \\
= & -\partial\left(\omega^{\bar{\partial} \dot{j}^{1,0}}\right)-(\partial \omega)^{\bar{\partial}^{j^{1,0}}}+(\partial \bar{\partial} \omega)^{j^{1,0}} \\
& -2 c\left(\bar{\partial} \dot{\theta}^{0,1}\right) \wedge F .
\end{aligned}
$$


The proof follows now using the third equation in (2.3).

Using Lemma 2.3, we go now for the proof of Proposition 2.3.

Proof of Proposition 2.3. We first prove that (2.31) is a complex. Note that $\mathbf{L} \circ \mathbf{P}=0$ implies $\widetilde{\mathbf{L}} \circ \mathbf{P}=0$ and trivially $d \circ \widetilde{\mathbf{d}}=0$. Hence, using that $C^{*}$ is a complex, it remains to prove that $\widetilde{\mathbf{d}} \circ \widetilde{\mathbf{L}}=0$. Given $(\dot{\Omega}, \dot{\theta}, \dot{\omega}) \in \tilde{A}^{1}$, using Lemma 2.3 we obtain

$$
\widetilde{\mathbf{d}} \circ \widetilde{\mathbf{L}}(\dot{\Omega}, \dot{\theta}, \dot{\omega})=\left(d\left(2 \operatorname{Re}\left(\partial\left(\omega^{\bar{\partial} \dot{j}^{1,0}}\right)\right)\right), 0\right),
$$

which vanishes for type reasons. We next prove that $\tilde{A}^{*}$ is elliptic. Ellipticity at steps one, two and five follows from ellipticity of the complex $C^{*}$ and the proof of Proposition 2.2. Ellipticity at step four follows from ellipticity of $C^{*}$ and of the complex

$$
\Omega^{2,2} \stackrel{d}{\longrightarrow} \Omega^{5} \stackrel{d}{\longrightarrow} \Omega^{6} \longrightarrow 0,
$$

combined with the formula for the symbol of $\widetilde{\mathbf{d}}$. We verified the ellipticity of $\tilde{A}^{*}$ at all steps but one, so it remains to show that an alternated sum of dimensions vanishes. Given a complex $B^{*}$ as above and $x \in M$, set $B_{x}^{j}$ to be the fiber at $x$ of the bundle on $M$ whose space of smooth global sections is $B^{j}$. With this notation, we need to show that

$$
\sum_{j}(-1)^{j} \operatorname{dim}\left(\tilde{A_{x}^{j}}\right)=0
$$

By (2.14),

and note that

$$
\tilde{A}^{1} \cong C^{1} \oplus \Omega_{\mathbb{R}}^{1,1}
$$

$$
\tilde{A}^{0} \oplus \Omega^{0} \cong C^{0} .
$$

By ellipticity of $C^{*}$, the following sum vanishes:

$$
\sum_{j}(-1)^{j} \operatorname{dim}\left(C_{x}^{j}\right)=0 .
$$

Then, by (2.39), (2.37) and (2.38), equation (2.36) is equivalent to

$$
\begin{aligned}
\operatorname{dim}\left(\Lambda^{0} T_{x}^{*}\right)= & -\operatorname{dim}\left(\Lambda_{\mathbb{R}}^{1,1} T_{x}^{*}\right)+\operatorname{dim}\left(\Lambda_{\mathbb{R}}^{2,2} T_{x}^{*} \oplus \Lambda^{5} T_{x}^{*} \oplus \Lambda^{6} T_{x}^{*}\right) \\
& -\operatorname{dim}\left(\Lambda^{5} T_{x}^{*} \oplus \Lambda^{6} T_{x}^{*}\right)+\operatorname{dim}\left(\Lambda^{6} T_{x}^{*}\right),
\end{aligned}
$$

which is trivially satisfied. Finally, by (2.32), $\widetilde{\mathbf{L}}=0$ is equivalent to $\mathbf{L}=0$, so the first cohomology of $\tilde{A}^{*}$ equals the cohomology of (2.17).

\section{Infinitesimal MOduli: General CASE}

In this section we construct the infinitesimal moduli space of solutions for the Strominger system. The main novelty with respect to the abelian setting is that the symmetries of the system must preserve the compatibility between the connection $\nabla$ and the U(3)-structure on the tangent bundle. As a result, the specific gauge transformations that we consider do not have a group structure, but nevertheless fit into the more general framework of Lie groupoid-actions. 
3.1. Notation and parameter space. Let $M$ be a compact, oriented, six dimensional smooth manifold. Let $P_{\mathrm{GL}^{+}}$be the principal $\mathrm{GL}^{+}(6, \mathbb{R})$-bundle of oriented frames of $M$. Let $P_{K}$ be a principal bundle, with compact structure group $K$. Let $P$ be the principal $G$-bundle given by the fiber product

$$
P=P_{K} \times{ }_{M} P_{\mathrm{GL}^{+}}
$$

with $G=K \times \mathrm{GL}^{+}(6, \mathbb{R})$. We fix a non-degenerate pairing on the Lie algebra

$$
\mathfrak{g}=\mathfrak{k} \oplus \mathfrak{g l}(6, \mathbb{R})
$$

of $G$, given by

$$
c=2 \alpha^{\prime}\left(-\operatorname{tr}_{\mathfrak{k}}-c_{\mathfrak{g l}}\right) .
$$

Here, $-\operatorname{tr}_{\mathfrak{k}}$ denotes the Killing form on $\mathfrak{k}$ and $c_{\mathfrak{g l}}$ is a non-degenerate invariant metric on $\mathfrak{g l}(6, \mathbb{R})$, which extends the non-degenerate Killing form - tr on $\mathfrak{s l}(6, \mathbb{R}) \subset \mathfrak{g l}(6, \mathbb{R})$.

Let $\mathcal{A}$ denote the space of product connections $\theta=A \times \nabla$ on $P$, where $A$ is a connection on $P_{K}$ and $\nabla$ is a connection on the tangent bundle $T$ of $M$. As in Section 2, we denote by $\Omega_{0}^{3} \subset \Omega_{\mathbb{C}}^{3}$ the space of complex 3-forms $\Omega$ such that (2.1) determines an almost complex structure $J_{\Omega}$ on $M$. For our analysis, we consider a parameter space

$$
\mathcal{P} \subset \Omega_{0}^{3} \times \mathcal{A} \times \Omega^{2}
$$

defined by

$$
\mathcal{P}=\left\{(\Omega, \theta, \omega) \mid \omega \text { is } J_{\Omega} \text { - compatible and } \nabla \text { is }\left(J_{\Omega}, \omega\right)-\text { unitary }\right\} .
$$

The points in $\mathcal{P}$ are regarded as unknowns for the system of equations

$$
\begin{aligned}
d \Omega & =0, & d\left(\|\Omega\|_{\omega} \omega^{2}\right)=0, \\
F_{\theta}^{0,2} & =0, & F_{\theta} \wedge \omega^{2}=0, \\
d d^{c} \omega-c\left(F_{\theta} \wedge F_{\theta}\right) & =0, &
\end{aligned}
$$

where $F_{\theta}$ denotes the curvature of $\theta=A \times \nabla$, given explicitly by

$$
F_{\theta}=F_{A}+R_{\nabla} \in \Omega^{2}(\operatorname{ad} P) .
$$

Our convention for the curvature tensor is [9]

$$
F_{\theta}=-\theta\left[\theta^{\perp} \cdot \theta^{\perp} \cdot\right] \in \Omega^{2}(\operatorname{ad} P),
$$

where $\theta$ is identified with a bundle morphism $\theta: T P \rightarrow V P, \theta^{\perp}:=\mathrm{Id}-\theta$ is the projection into the horizontal subspace and the Bracket denotes the Lie bracket of vector fields on $P$. Alternatively, we will use the formula

$$
F_{\theta}=d \theta+\frac{1}{2}[\theta, \theta]
$$

where $\theta$ is regarded as a $G$-invariant 1 -form in $P$ with values in $\mathfrak{g}$ and the bracket is the one on the Lie algebra. The induced covariant derivative on the bundle of Lie algebras ad $P=P \times_{G} \mathfrak{g}$ is

$$
i_{V} d^{\theta} r=\left[\theta^{\perp} V, r\right]
$$

which satisfies $d^{\theta} \circ d^{\theta}=\left[F_{\theta}, \cdot\right]$. 
Remark 3.1. The compatibility between $\nabla$ and the SU(3)-structure on $M$ is unmotivated from the physics point of view, but it is helpful for the mathematics that follow, making our discussion more standard. For the physics of the Strominger system it is required the weaker assumption that $\nabla$ is compatible with the metric underlying the $\mathrm{SU}(3)$-structure.

Solutions of (3.2) are in correspondence with structures of Calabi-Yau manifold on $M$, endowed with a solution of the Strominger system (1.1), as it follows from

$$
c\left(F_{\theta} \wedge F_{\theta}\right)=\alpha^{\prime}\left(\operatorname{tr} R_{\nabla} \wedge R_{\nabla}-\operatorname{tr}_{\mathfrak{k}} F_{A} \wedge F_{A}\right) .
$$

Note here that the compatibility between $\nabla$ and $\left(J_{\Omega}, \omega\right)$ reduces the holonomy of $\nabla$ to $\mathrm{U}(3) \subset \mathrm{SL}(6, \mathbb{R})$, and $c_{\mid \mathfrak{s}(6, \mathbb{R})}=-\operatorname{tr}$.

We now proceed to the description of the tangent space of $\mathcal{P}$. Fix an element $(\Omega, \theta, \omega)$ of $\mathcal{P}$ and set $T_{0} \mathcal{P}$ to be the tangent space of $\mathcal{P}$ at this point. Differentiating the compatibility conditions $\nabla J=0$ and $\nabla \omega=0$, we obtain

$$
\nabla \dot{J}+[\dot{\nabla}, J]=0, \quad \nabla \dot{\omega}-\omega^{\dot{\nabla}}=0,
$$

where $\dot{\nabla} \in \Omega^{1}(\operatorname{End} T)$ is the variation of $\nabla$ and

$$
\omega^{\dot{\nabla}}(X, Y)=\omega(\dot{\nabla} X, Y)+\omega(X, \dot{\nabla} Y)
$$

for all $X, Y \in \Omega^{0}(T)$. Then, the non skew-hermitian part of $\dot{\nabla}$ with respect to $\left(J_{\Omega}, \omega\right)$ is determined by the variations $\dot{J}$ and $\dot{\omega}$ and we find an isomorphism

$$
T_{0} \mathcal{P} \cong S_{1}:=\Omega^{3,0} \oplus \Omega^{2,1} \oplus \Omega^{1}\left(\operatorname{ad} P_{h}\right) \oplus \Omega_{\mathbb{R}}^{1,1},
$$

where

$$
P_{h}=P_{K} \times_{M} P_{\mathrm{U}(3)},
$$

and $P_{\mathrm{U}(3)} \subset P_{\mathrm{GL}^{+}}$is the $=\mathrm{U}(3)$-reduction determined by the bundle of unitary frames of $\left(J_{\Omega}, \omega\right)$. This isomorphism is explicitly defined combining (2.15) and

$$
\dot{\nabla}=\dot{\nabla}_{h}-\nabla\left(\frac{1}{2} J \dot{J}+\frac{1}{4}(\dot{\omega} J+J \dot{\omega})\right)
$$

for $\dot{\nabla}_{h} \in \Omega^{1}\left(\right.$ ad $\left.P_{\mathrm{U}(3)}\right)$. Note that in (3.5) $), \dot{\omega}$ stands for the element of $\Omega^{0}(\operatorname{End}(T))$ associated to $\dot{\omega}$ via the metric $g=\omega(\cdot, J \cdot)$. We also note that, unlike (2.14), the isomorphism $S_{1} \rightarrow T_{0} \mathcal{P}$ is a differential operator of order 1 , and hence it is not induced by a bundle isomorphism. This will be important in what follows.

3.2. Gauge groupoid and infinitesimal action. We define now the symmetries that we will use to construct the space of infinitesimal variations of the Strominger system. Given $g \in$ Aut $P$, we denote by $\check{g} \in$ Diff the diffeomorphism in the base that it covers. Consider the groupoid

$$
\widetilde{\mathcal{G}} \rightrightarrows \mathcal{P}
$$

given by

$$
\widetilde{\mathcal{G}}:=\left\{(g, \Omega, \theta, \omega) \in \operatorname{Aut} P \times \mathcal{P} \mid g^{*}\left(J_{\Omega}, \omega\right)=\check{g}^{*}\left(J_{\Omega}, \omega\right)\right\},
$$

with source and target maps

$$
s(g, x)=x, \quad t(g, x)=x g,
$$


where $x \in \mathcal{P}$ and the right action is given by pull-back. We refer to [54 for basic definitions on groupoids. The proof of the following result is straightforward and is therefore omitted.

Lemma 3.1. The action of $\widetilde{\mathcal{G}}$ on $\mathcal{P}$ preserves (3.2), that is, if $x \in \mathcal{P}$ is a solution of (3.2) then $x g$ is also a solution for all $(g, x) \in s^{-1}(x)$.

We fix an element $(\Omega, \theta, \omega) \in \mathcal{P}$ and denote $F=F_{\theta}, R=R_{\nabla}$ and $J=J_{\Omega}$. There is a natural bijection

$$
s^{-1}(\Omega, \theta, \omega) \cong \operatorname{Aut} P_{K} \times \mathcal{G}_{\mathrm{U}(3)},
$$

where $\mathcal{G}_{\mathrm{U}(3)}$ denotes the gauge group of the $\mathrm{U}(3)$-structure $(J, \omega)$. Hence, using the connection $A$ on $P_{K}$, the vector space of infinitesimal symmetries is given by

$$
S^{0}=\Omega^{0}(T) \oplus \Omega^{0}\left(\operatorname{ad} P_{h}\right) .
$$

Lemma 3.2. The infinitesimal action of $(V, t) \in \Omega^{0}(T) \oplus \Omega^{0}\left(\operatorname{ad} P_{\mathrm{U}(3)}\right)$ on $\nabla$ is given by

$$
d^{\nabla} t+d^{\nabla}(\nabla V)+i_{V} R
$$

where $d^{\nabla}(\nabla V)$ denotes the exterior derivative $d^{\nabla}$ on $\Omega^{*}($ End $T)$, induced by $\nabla$, acting on the endomorphism $\nabla V \in \Omega^{0}(\operatorname{End} T)$.

Proof. For an arbitrary element $\zeta \in$ Lie Aut $P_{\mathrm{GL}^{+}}$we can write uniquely

$$
\zeta=t+\nabla^{\perp} V
$$

for suitable $t \in \Omega^{0}\left(\operatorname{ad} P_{\mathrm{GL}^{+}}\right)$and $V \in \Omega^{0}(T)$, where $\nabla^{\perp} V$ denotes the horizontal lift of $V$ with respect to $\nabla$. Then, using this splitting, the infinitesimal action of $\zeta$ on $\nabla$ reads

$$
\zeta \cdot \nabla=d^{\nabla} t+i_{V} R
$$

Here we note that ad $P_{\mathrm{GL}^{+}}=$End $T$ and $\nabla t$ denotes the covariant derivative on End $T$ acting on the endomorphism $t \in \Omega^{0}($ End $T)$. Finally, given $V \in \Omega^{0}(T)$, we note that $d V: T \rightarrow T(T)$ is the image of $V$ by the Lie algebra morphism

$$
\Omega^{0}(T) \rightarrow \text { Lie Aut } P_{\mathrm{GL}^{+}}
$$

induced by the natural inclusion $0 \rightarrow \operatorname{Diff}_{0} \rightarrow$ Aut $P_{\mathrm{GL}^{+}}$, and hence the result follows from the formula

$$
\nabla V=d V-\nabla^{\perp} V
$$

By Lemma 3.2, the infinitesimal action of $\widetilde{\mathcal{G}}$ on $(\Omega, \theta, \omega) \in \mathcal{P}$ reads:

$$
(V, r) \cdot(\Omega, \theta, \omega)=\left(d \iota_{V} \Omega,\left(r+\theta^{\perp} V\right) \cdot \theta, \mathcal{L}_{V} \omega\right),
$$

where $r=s+t \in \Omega^{0}\left(\operatorname{ad} P_{K} \oplus \operatorname{ad} P_{\mathrm{U}(3)}\right)$ and

$$
\left(r+\theta^{\perp} V\right) \cdot \theta=\left(d^{A} s+\iota_{V} F_{A}, d^{\nabla} t+d^{\nabla}(\nabla V)+i_{V} R\right) .
$$

Here, $d^{A}$ denotes the exterior derivative induced by $A$ on $\Omega^{*}\left(\operatorname{ad} P_{K}\right)$. Using the previous formula and the isomorphism (3.4), we define a differential operator

$$
\begin{array}{cccc}
\mathbf{P}: & S^{0} & \rightarrow & S^{1} \\
(V, r) & \mapsto & \left(d \iota_{V} \Omega, \dot{\theta}_{h},\left(\mathcal{L}_{V} \omega\right)^{1,1}\right),
\end{array}
$$


MODULI, STROMINGER AND KILLING SPINORS IN GENERALIZED GEOMETRY 19 where

$$
\dot{\theta}_{h}=\left(r+\theta^{\perp} V\right) \cdot \theta+\nabla\left(\frac{1}{2} J \dot{J}+\frac{1}{4}(\dot{\omega} J+J \dot{\omega})\right) \in \Omega^{1}\left(\operatorname{ad} P_{h}\right),
$$

for $\dot{\omega}=\mathcal{L}_{V} \omega$ and $\dot{J}$ given by (2.12). We conclude this section with the following lemma, whose proof is left to the reader.

Lemma 3.3. The tuples $\mathbf{t}=(2,1,1)$ and $\mathbf{s}=(1,0,0,1)$ form a system of orders for $\mathbf{P}$ and the associated leading symbol is given by

$$
\sigma_{\mathbf{P}}(v)(V, r)=\left(v \wedge \iota_{V} \Omega, v \otimes s, v \otimes\left(t+(v \otimes V)_{h}\right),\left(v \wedge \iota_{V} \omega\right)^{1,1}\right),
$$

where $v \in T^{*} \backslash M$ and $(v \otimes V)_{h}$ is the skew-hermitian part of the endomorphism $v \otimes V$.

3.3. Linearization and ellipticity. Let $(\Omega, \theta, \omega) \in \mathcal{P}$ be a solution of (3.2). Let us denote by $\mathbf{L}$ the differential at $(\Omega, \theta, \omega)$ of (3.2) with respect to variations in $T_{0} \mathcal{P}$. We set

$$
S^{2}=\Omega^{3,1} \oplus \Omega^{2,2} \oplus \Omega^{0,2}\left(\operatorname{ad} P_{h}\right) \oplus \Omega^{4} \oplus \Omega^{5} \oplus \Omega^{6}\left(\operatorname{ad} P_{h}\right) .
$$

Lemma 3.4. The operator $\mathbf{L}$ takes values in $S^{2}$ and induces an operator

$$
\mathbf{L}=\oplus_{i=1}^{5} \mathbf{L}_{i}: S^{1} \rightarrow S^{2}
$$

via (3.4), given by

$$
\begin{aligned}
& \mathbf{L}_{1}\left(\dot{\Omega}, \dot{\theta}_{h}, \dot{\omega}^{1,1}\right)=d \dot{\Omega}, \\
& \mathbf{L}_{2}\left(\dot{\Omega}, \dot{\theta}_{h}, \dot{\omega}^{1,1}\right)=\bar{\partial}^{\theta}\left(\dot{\theta}_{h}\right)^{0,1}+\frac{i}{2}\left(F^{\dot{J}}\right)^{0,2}, \\
& \mathbf{L}_{3}\left(\dot{\Omega}, \dot{\theta}_{h}, \dot{\omega}^{1,1}\right)=d\left(J d \dot{\omega}-J(d \omega)^{\dot{J} J}-2 c\left(\dot{\theta}_{h} \wedge F\right)\right), \\
& \mathbf{L}_{4}\left(\dot{\Omega}, \dot{\theta}_{h}, \dot{\omega}^{1,1}\right)=d\left(2\|\Omega\|_{\omega} \dot{\omega} \wedge \omega+\delta\left(\|\Omega\| \|_{\omega}\right) \omega^{2}\right), \\
& \mathbf{L}_{5}\left(\dot{\Omega}, \dot{\theta}_{h}, \dot{\omega}^{1,1}\right)=d^{\theta} \dot{\theta}_{h} \wedge \omega^{2}+2 F \wedge \dot{\omega} \wedge \omega,
\end{aligned}
$$

where $\dot{\omega}=\dot{\omega}^{1,1}+\frac{1}{2} \dot{\omega}^{j J}, \dot{J}$ is the infinitesimal variation of almost-complex structure (2.12), $\delta\left(\|\Omega\|_{\omega}\right)$ is given by formula (2.19), and $\bar{\partial}^{\theta}=p^{(0,1)} \circ d^{\theta}$ is the Dolbeault operator induced by $\theta$.

Proof. The computations follow as in the proof of Lemma 2.1. The crucial step is to show that the only contribution to $\mathbf{L}: T_{0} \mathcal{P} \rightarrow S^{2}$ in the variation of $F$

$$
d^{\theta} \dot{\theta}=d^{A} \dot{A}+d^{\nabla} \dot{\nabla}
$$

comes from $d^{A} \dot{A}+d^{\nabla} \dot{\nabla}_{h}$, where $\dot{\theta}=\dot{A}+\dot{\nabla}$ and $\dot{\theta}_{h}=\dot{A}+\dot{\nabla}_{h}$ (see (3.5)). To simplify the exposition, for a moment we denote

$$
q=\frac{1}{2} J \dot{J}+\frac{1}{4}(\dot{\omega} J+J \dot{\omega})
$$

so that

$$
\dot{\nabla}=\dot{\nabla}_{h}-\nabla q
$$


With this notation,

$$
\begin{aligned}
d^{\nabla} \dot{\nabla} & =d^{\nabla} \dot{\nabla}_{h}-d^{\nabla} d^{\nabla} q \\
& =d^{\nabla} \dot{\nabla}_{h}-[R, q] .
\end{aligned}
$$

Then, as $(\Omega, \theta, \omega)$ is a solution of (3.2),$R^{0,2}=0$ and $R \wedge \omega^{2}=0$, and therefore

$$
\begin{aligned}
{[R, q]^{0,2} } & =0, \\
{[R, q] \wedge \omega^{2} } & =0 .
\end{aligned}
$$

Jointly with (3.15), formulae for $\mathbf{L}_{2}$ and $\mathbf{L}_{5}$ follow. Finally, to calculate $\mathbf{L}_{3}$, we note that

$$
\operatorname{tr}([R, q] \wedge R)=0
$$

Consider the following complex of differential operators

$$
S^{0} \stackrel{\mathbf{P}}{\longrightarrow} S^{1} \stackrel{\mathbf{L}}{\longrightarrow} S^{2} .
$$

Theorem 3.1. The complex (3.16) is elliptic. The space of infinitesimal deformations of solutions to the Strominger system of equations is defined as the first cohomology group $H^{1}\left(S^{*}\right)$.

Proof. As the $\widetilde{\mathcal{G}}$-action preserves solutions of (3.2), $\mathbf{L} \circ \mathbf{P}=0$. Assume that $\sigma_{\mathbf{L}}(v)\left(\dot{\Omega}, \dot{\theta}_{h}, \dot{\omega}^{1,1}\right)=0$, with $\dot{\theta}_{h}=\dot{A}+\dot{\nabla}_{h}$ (see (3.14) $)$. Then, arguing as in the proof of Proposition 2.2 we obtain

$$
\left(\dot{\Omega}, \dot{A}, \dot{\nabla}_{h}, \dot{\omega}^{1,1}\right)=\left(v \wedge \iota_{V} \Omega, v \otimes s, v \otimes t,\left(v \wedge \iota_{V} \omega\right)^{1,1}\right)
$$

for some $V \in T$ and $s+t \in$ ad $P_{h}$. Trivially,

$$
\dot{\nabla}_{h}=v \otimes\left(t^{\prime}+(v \otimes V)_{h}\right)
$$

for $t^{\prime} \in \operatorname{ad} P_{h}$ and hence the result follows.

We finish this section extending the complex (3.16), in order to define a space of obstructions to integrability for infinitesimal deformations of the Strominger system. We follow closely the method in Section 2.4. Define

$$
\begin{aligned}
\tilde{S}^{0} & :=S^{0}, \\
\tilde{S}^{1} & :=S^{1}, \\
\tilde{S}^{2} & :=\Omega^{3,1} \oplus \Omega^{2,2} \oplus \Omega^{0,2}\left(\operatorname{ad} P_{h}\right) \oplus \Omega_{\mathbb{R}}^{2,2} \oplus \Omega^{5} \oplus \Omega^{6}\left(\operatorname{ad} P_{h}\right), \\
\tilde{S^{3}} & :=\Omega^{3,2} \oplus \Omega^{2,3} \oplus \Omega^{0,3}\left(\operatorname{ad} P_{h}\right) \oplus \Omega^{5} \oplus \Omega^{6}, \\
\tilde{S^{4}} & :=\Omega^{3,3} \oplus \Omega^{6},
\end{aligned}
$$

and

$$
0 \longrightarrow \tilde{S}^{0} \stackrel{\mathbf{P}}{\longrightarrow} \tilde{S}^{1} \stackrel{\widetilde{\mathbf{L}}}{\longrightarrow} \tilde{S}^{2} \stackrel{\bar{\partial}_{0} \oplus \widetilde{\mathbf{d}}}{\longrightarrow} \tilde{S}^{3} \stackrel{\bar{\partial}_{0} \oplus d}{\longrightarrow} \tilde{S}^{4} \longrightarrow 0 .
$$

Here

$$
\bar{\partial}_{0}(\alpha, \beta)=\left(d \alpha, \bar{\partial}^{\theta} \beta-F^{T_{0}^{-1}\left(\alpha^{2, j}\right)}\right) .
$$


for $\alpha \in \Omega^{3, j-1} \oplus \Omega^{2, j}$ and $\beta \in \Omega^{0, j}\left(\operatorname{ad} P_{h}\right)$, for $j=2,3$, and

$$
\widetilde{\mathbf{d}}(\alpha, \beta, \gamma, \delta, \epsilon)=\left(d\left(\gamma-4 \operatorname{Re}\left(i(\partial \omega)^{T_{0}^{-1}\left(\alpha^{2,2}\right)}+\frac{1}{2} c(\beta \wedge F)\right)\right), d \delta\right) .
$$

The proof of the following result follows easily combining the proof of Proposition 2.3 with the ellipticity of Kim's complex [44] (see also [45, p. 246]).

Theorem 3.2. The complex $\tilde{S}^{*}$ is elliptic and there is a natural isomorphism $H^{1}\left(\tilde{S}^{*}\right)=H^{1}\left(S^{*}\right)$. The space of obstructions to integrability of infinitesimal deformations of the Strominger system of equations is defined as the second cohomology group $H^{2}\left(\tilde{S}^{*}\right)$ of $\tilde{S}^{*}$.

\section{AnOmaly CANCELlation, Flux QUANTIZATiOn AND GENERALIZED GEOMETRY}

The aim of this section is to initiate the study of the geometry of the moduli space of solutions of the Strominger system which emerges from the Bianchi identity. We describe the infinitesimal structure of a natural foliation in the moduli space, whose leaves are intimately related to generalized geometry.

4.1. An integrable distribution in the moduli space. This prelude intends to serve as motivation for the rest of this section. Our discussion is to be taken rather formally, since we do not want to get involved here with the differential-topological aspects of the problem. In particular, the construction of a natural differentiable structure on the moduli space of solutions of the Strominger system will be addressed in a sequel of the present work.

Following the notation of Section 3 , the moduli space of solutions of the Strominger system can be identified with the quotient

$$
\mathcal{M}=\mathcal{P}_{S} / \widetilde{\mathcal{G}}
$$

where $\mathcal{P}_{S} \subset \mathcal{P}$ is the locus where the equations (3.2) are satisfied. Using the transgression formula for the Chern-Simons 3 -form, given any point $(\Omega, \theta, \omega)$ in $\mathcal{M}$ we define a $H^{3}(M, \mathbb{R})$-valued function

$$
\vartheta\left(\Omega^{\prime}, \theta^{\prime}, \omega^{\prime}\right)=\left[d^{c^{\prime}} \omega^{\prime}-d^{c} \omega-2 c(a \wedge F)-c\left(a \wedge d^{\theta} a\right)-\frac{1}{3} c(a \wedge[a, a])\right],
$$

where $\theta^{\prime}=\theta+a$. This function is well-defined up to the action of the equivariant mapping class group of the principal bundle $P$ and hence it defines a global, closed, $H^{3}(M, \mathbb{R})$-valued 1-form

$$
\delta \in \Omega^{1}\left(\mathcal{M}, H^{3}(M, \mathbb{R})\right),
$$

induced by the explicit expression

$$
\delta(\dot{\Omega}, \dot{\theta}, \dot{\omega})=\left[J d \dot{\omega}-J(d \omega)^{\dot{J} J}-2 c\left(\dot{\theta}_{h} \wedge F\right)\right] .
$$

Neglecting obstructions to integrability, the tangent space of $\mathcal{M}$ at a point can be identified with the cohomology group $H^{1}\left(S^{*}\right)$ and the closed 1-form $\delta$ induces now an integrable distribution

$$
0 \longrightarrow \operatorname{Ker} \delta \longrightarrow H^{1}\left(S^{*}\right) \stackrel{\delta}{\longrightarrow} H^{3}(M, \mathbb{R})
$$


and therefore a foliation on the moduli space. The leaves of this foliation are closely related to generalized geometry, as we will rigorously show in this section at the infinitesimal level.

Motivation for our construction comes from the Green-Schwarz mechanism [34] and the flux quantization condition, two basic principles in the physics of the heterotic string. On the one hand, physics claims that the fundamental equation relating the unitary structure on the manifold with the pair of connections $\nabla$ and $A$ is a local equation for 3 -forms, the so-called Green-Schwarz anomaly cancellation condition (or anomaly equation, for short)

$$
H=d b-\alpha^{\prime}(C S(\nabla)-C S(A)),
$$

written in terms of the Chern-Simons 3-forms of $A$ and $\nabla$, where $H:=d^{c} \omega$ is the 3-form flux. This requires the introduction of an additional ingredient: a local real 2-form $b$-usually known as B-field potential. The Bianchi identity (1.3) is obtained by taking the exterior derivative in (4.1). On the other hand, the second principle says that the 'closed part' of the flux $H$, obtained by gluing the local closed 3 -forms $d b$, is quantized (see e.g. [3, 17]), giving a fixed integral cohomology class in $H^{3}(M, \mathbb{Z})$. Although the Green-Schwarz mechanism can be rigorously understood in topological grounds using the theory of differential string structures [62, here we shall focus on its interpretation in terms of geometry in the moduli space. In this setup, level sets of the multi-valued function $\vartheta$ correspond formally to solutions of the anomaly equation (4.1), while the kernel of the 1 -form $\delta$ can be interpreted as infinitesimal variations which preserve the flux quantization.

4.2. Constraining infinitesimal variations: abelian case. To simplify the exposition and highlight the main ideas, we start our discussion with the abelian case studied in Section 2. Let $H^{1}\left(A^{*}\right)$ be the finite-dimensional vector space of infinitesimal variations of the given solution $(\Omega, \theta, \omega)$, constructed in Section 2. We note from (2.18) that there is a natural linear map

$$
\delta: H^{1}\left(A^{*}\right) \rightarrow H^{3}(M, \mathbb{R})
$$

given by

$$
[(\dot{\Omega}, \dot{\theta}, \dot{\omega})] \mapsto\left[J d \dot{\omega}-J(d \omega)^{\dot{j} J}-2 c(\dot{\theta} \wedge F)\right]
$$

and we denote by $H^{1}\left(\AA^{*}\right):=\operatorname{Ker} \delta$. For an element $[(\dot{\Omega}, \dot{\theta}, \dot{\omega})]$ of $H^{1}\left(\AA^{*}\right)$, the equation $\mathbf{L}_{3}((\dot{\Omega}, \dot{\theta}, \dot{\omega}))=0$ is satisfied as a consequence of the stronger condition

$$
J d \dot{\omega}-J(d \omega)^{\dot{J} J}-2 c(\dot{\theta} \wedge F)=d b,
$$

for a 2 -form $b \in \Omega^{2}$.

Being a fundamental parameter space, we would like to identify precisely the objects that $H^{1}\left(\AA^{*}\right)$ parameterizes. In a first approximation, it is natural to ask whether $H^{1}\left(\AA^{*}\right)$ arises naturally as the cohomology of a complex of geometric origin. We give next an affirmative answer to this preliminary question, but somehow in an unexpected way: to construct the complex, we need to enlarge the first two vector spaces in (2.17) (or (2.31)) by adding the space of 2forms $\Omega^{2}$. The 2-forms become part of the symmetries of the problem, but 
also contribute as additional data for the geometric objects parameterized by $H^{1}\left(\stackrel{\circ}{A}^{*}\right)$. Consider the following sequence

$$
A^{0} \oplus \Omega^{2} \stackrel{\stackrel{\circ}{\mathbf{P}}}{\longrightarrow} A^{1} \oplus \Omega^{2} \stackrel{\stackrel{\circ}{\mathbf{L}}}{\longrightarrow} \stackrel{\circ}{\mathcal{R}},
$$

where we follow the notation of Section 2.4. Here,

$$
\mathcal{R}:=\Omega^{3,1} \oplus \Omega^{2,2} \oplus \Omega^{0,2}(i \mathbb{R}) \oplus \Omega^{3} \oplus \Omega^{5} \oplus \Omega^{6}(i \mathbb{R}),
$$

and the maps $\stackrel{\circ}{\mathbf{P}}$ and $\stackrel{\circ}{\mathbf{L}}$ are defined by

$$
\stackrel{\circ}{\mathbf{P}}(V, r, B)=(\mathbf{P}(V, r),-B)
$$

and

$$
\stackrel{\circ}{\mathbf{L}}=\mathbf{L}_{1} \oplus \mathbf{L}_{2} \oplus \stackrel{\circ}{\mathbf{L}}_{3} \oplus \mathbf{L}_{4} \oplus \mathbf{L}_{5}
$$

where

$$
\stackrel{\circ}{\mathbf{L}}_{3}(\dot{\Omega}, \dot{\theta}, \dot{\omega}, b)=J d \dot{\omega}-J(d \omega)^{\dot{J} J}-2 c(\dot{\theta} \wedge F)-d b .
$$

The main difference with (2.17) is that we have decreased by one the degree of the original map $\mathbf{L}_{3}$ in such a way that $\stackrel{\circ}{\mathbf{L}}_{3}=0$ is precisely the equation (4.3). Unlike (2.17), equation (4.4) is a sequence of differential operators of degree 1, but it is not a complex.

Lemma 4.1. The sequence (4.4) defines a complex by restriction of the first arrow to elements $(V, r, B) \in A^{0} \oplus \Omega^{2}$ satisfying the equation

$$
\mathcal{L}_{V}\left(d^{c} \omega\right)=2 c\left(\left(d r+\iota_{V} F\right) \wedge F\right)-d B .
$$

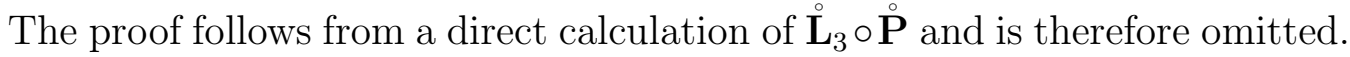
Note that using the Bianchi identity (last equation in (2.3)), we can rewrite (4.5) as

$$
d\left(i_{V} d^{c} \omega-2 c r F\right)=-d B .
$$

A fact worth mentioning is that the space of $(V, r, B)$ satisfying (4.5) has a natural Lie algebra structure. We will come back to this in Section 4.5. By now, we use the suggestive notation

$$
\text { Lie Aut } E:=\left\{(V, r, B) \in A^{0} \oplus \Omega^{2} \text { satisfying (4.5) }\right\} \text {. }
$$

Lemma 4.2. The cohomology of the complex of vector spaces

$$
\text { Lie Aut } E \stackrel{\stackrel{\circ}{\mathbf{P}}}{\longrightarrow} A^{1} \oplus \Omega^{2} \stackrel{\stackrel{\circ}{\longrightarrow}}{\longrightarrow} \mathcal{R}
$$

is naturally isomorphic to $H^{1}\left(\stackrel{\circ}{ }^{*}\right)$. Consequently, the cohomology of $\AA^{*}$ is finite dimensional.

Proof. For a moment, denote by $H^{1}$ the cohomology of (4.8). Then, we have a natural linear map $\Phi: H^{1} \rightarrow H^{1}\left(\AA^{*}\right)$ given by sending a class $[(\dot{\Omega}, \dot{\theta}, \dot{\omega}, b)]$ in $H^{1}$ to the class $[(\dot{\Omega}, \dot{\theta}, \dot{\omega})]$ in $H^{1}\left(\AA^{*}\right)$. By definition of $H^{1}\left(\AA^{*}\right)$, this map is trivially surjective. Assume now that $[(\dot{\Omega}, \dot{\theta}, \dot{\omega}, b)] \in H^{1}$ is such that $[(\dot{\Omega}, \dot{\theta}, \dot{\omega})]=0$ in $H^{1}\left(\AA^{*}\right)$. Then, $(\dot{\Omega}, \dot{\theta}, \dot{\omega})=\mathbf{P}(V, r)$ and from the condition

$$
\stackrel{\circ}{\mathbf{L}}_{3}(\mathbf{P}(V, r), b)=0
$$

it follows that $(V, r, b) \in$ Lie Aut $E$, proving that the map $\Phi$ is injective. 
Note that the complex (4.8) is not a complex of differential operators in the standard sense. This is due to the fact that Lie Aut $E$ is described by the differential equation (4.5) and hence it does not correspond to the space of sections of any vector bundle. Therefore, the theory of elliptic operators does not apply to (4.8), and there is no direct way of proving that its cohomology is finite dimensional. We obtain this result by comparison with the elliptic complex (2.17).

4.3. Weakening the symmetries: the elliptic complex $\widehat{A}^{*}$. Leaving aside for a moment the geometric interpretation of the parameter space $H^{1}\left(\AA^{*}\right)$, we would like to construct a related complex $\widehat{A}^{*}$ which is, unlike $\AA^{*}$, an elliptic complex of differential operators in the standard sense. Furthermore, we will construct $\widehat{A}^{*}$ using degree 1 operators, and therefore simplifying the complex $A^{*}$. The cohomology of this new complex, constructed by weakening the symmetries of $\stackrel{\circ}{A}^{*}$, will fit into an exact diagram of linear maps

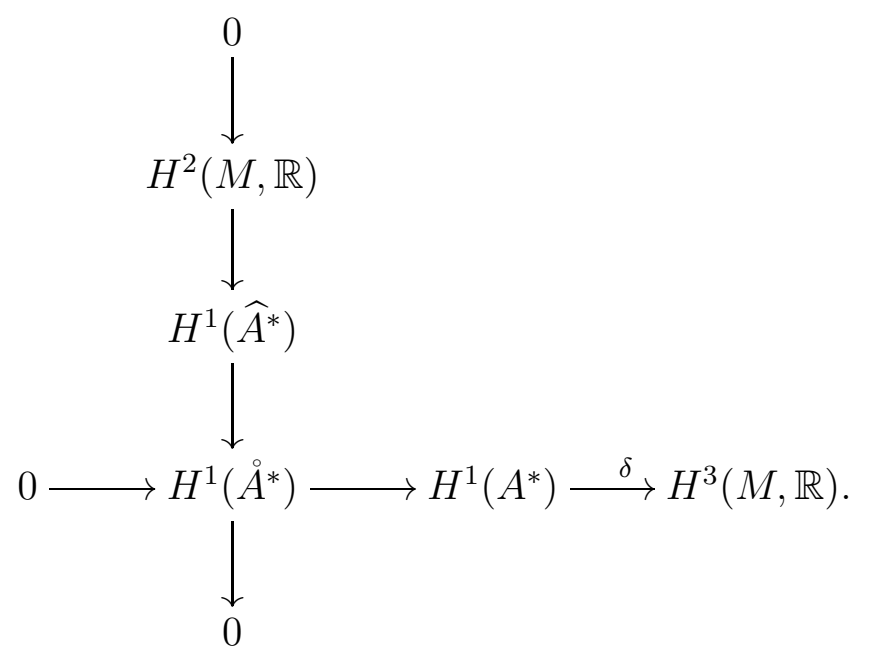

In the way, we will pin down some of the additional ingredients that we need to describe $H^{1}\left(\stackrel{\circ}{A}^{*}\right)$. Consider the vector bundle

$$
E=T \oplus i \mathbb{R} \oplus T^{*}
$$

and note that $\Omega^{0}(E):=A^{0} \oplus \Omega^{1}$. We define a sequence of differential operators

$$
\left(\widehat{A}^{*}\right) \quad \Omega^{0}(E) \stackrel{\stackrel{\circ}{\mathbf{P}}}{\longrightarrow} A^{1} \oplus \Omega^{2} \stackrel{\stackrel{\circ}{\mathbf{L}}}{\longrightarrow} \mathcal{R},
$$

where the map $\stackrel{\circ}{\mathbf{P}}$ is now defined by

$$
\stackrel{\circ}{\mathbf{P}}(V, r, \xi)=\left(\mathbf{P}(V, r), d \xi+i_{V}\left(d^{c} \omega\right)-2 c r F\right) .
$$

A straightforward calculation shows that

$$
\left(V, r,-d \xi-i_{V}\left(d^{c} \omega\right)+2 c r F\right) \in \text { Lie Auit } E
$$

and therefore (4.9) is a complex of differential operators. 
Proposition 4.1. The sequence (4.9) is an elliptic complex of differential operators of degree 1 . There is an exact sequence

$$
0 \rightarrow H^{2}(M, \mathbb{R}) \rightarrow H^{1}\left(\widehat{A}^{*}\right) \rightarrow H^{1}\left(\AA^{*}\right) \rightarrow 0
$$

where $H^{1}\left(\widehat{A}^{*}\right)$ denotes the cohomology of (4.9)).

Proof. By ellipticity of (2.17), $\sigma_{\mathbf{L}}(\dot{\Omega}, \dot{\theta}, \dot{\omega}, b)=0$ implies $(\dot{\Omega}, \dot{\theta}, \dot{\omega})=\sigma_{\dot{\mathbf{P}}}(V, r)$. In addition,

$$
0=\sigma_{\dot{L}_{3}}(v)(\dot{\Omega}, \dot{\theta}, \dot{\omega}, b)=J\left(v \wedge v \wedge i_{V} \omega\right)-v \wedge b=-v \wedge b
$$

and therefore $b=v \wedge v^{\prime}$ by ellipticity of the De Rham complex, proving ellipticity of (4.9).

The linear map $\Phi$ which sends a class $[(\dot{\Omega}, \dot{\theta}, \dot{\omega}, b)] \in H^{1}\left(\widehat{A}^{*}\right)$ to the class of $(\dot{\Omega}, \dot{\theta}, \dot{\omega}, b)$ in $H^{1}\left(\AA^{*}\right)$ is trivially well-defined and surjective. Assume now that the class of $(\dot{\Omega}, \dot{\theta}, \dot{\omega}, b)$ is zero in $H^{1}\left(\stackrel{\circ}{A}^{*}\right)$. Then, $(\dot{\Omega}, \dot{\theta}, \dot{\omega})=\mathbf{P}(V, r)$ and from the condition $\stackrel{\circ}{\mathbf{L}}_{3}(\mathbf{P}(V, r), b)=0$ and (4.6) it follows that

$$
d\left(b-i_{V}\left(d^{c} \omega\right)+2 c r F\right)=0 .
$$

We can construct a map $\operatorname{Ker} \Phi \rightarrow H^{2}(M, \mathbb{R})$, defined by

$$
[(\mathbf{P}(V, r), b)] \mapsto\left[b-i_{V}\left(d^{c} \omega\right)+2 c r F\right],
$$

which is an isomorphism.

Remark 4.1. Note the strong analogy between $\Omega^{0}(E)$ and Lie Aut $E$ and, respectively, the Hamiltonian vector fields and the symplectic vector fields on a symplectic manifold. In fact, the natural map defined by (4.10) defines an exact sequence

$$
0 \rightarrow \Omega^{0}(E) \rightarrow \text { Lie Auit } E \rightarrow H^{2}(M, \mathbb{R}) \rightarrow 0,
$$

which provides an analogue for the (differential of the) flux map in symplectic geometry. In the next section, we will see that Lie Aut $E$ has the structure of a Lie algebra while $\Omega^{0}(E)$ can be endowed with the structure of a Courant algebra.

4.4. Relation with generalized geometry of type $B_{n}$. The construction in Section 4.2 and Section 4.3 may look rather strange from a classical perspective, but turns out to be very natural in generalized geometry. The precise theoretical framework that we need was introduced by Baraglia [5] and developed by the second author [61], and goes under the name of generalized geometry of type $B_{n}$.

To explain the most basic aspects of this relation, in this section we consider the vector bundle

$$
E=T \oplus i \mathbb{R} \oplus T^{*}
$$

with an additional structure - the one of a smooth Courant algebroid. This bundle is such that its space of global sections $\Omega^{0}(E)$ provides the infinitesimal symmetries for the elliptic complex (4.9) and the Lie algebra of infinitesimal automorphisms of the Courant algebroid structure contains Lie Aut $E$ as a Lie subalgebra. In addition, the space of parameters $A^{1} \oplus \Omega^{2}$ - the middle 
step in the complexes (4.8) and (4.9) - will be naturally interpreted as a space of infinitesimal variations of a generalized metric on $E$. The explanation of more intricate aspects of our construction in terms of generalized geometry is postponed to Section 5 .

We fix our solution $(\Omega, \theta, \omega)$ of the abelian system (2.3). We shall emphasize that the rest of this section relies solely on having a solution of the abelian Bianchi identity

$$
d H=c(F \wedge F)
$$

with $H=d^{c} \omega$ and does not use the other structures provided by the equations. Consider the smooth vector bundle (4.11) endowed with the symmetric pairing

$$
\langle X+r+\xi, Y+t+\eta\rangle=\frac{1}{2}(\eta(X)+\xi(Y))+c(r t)
$$

and the canonical projection

$$
\pi: E \rightarrow T
$$

Using the quantities $H$ and $F$, we can endowed $\Omega^{0}(E)$ with a Dorfman bracket

$$
\begin{aligned}
{[X+r+\xi, Y+t+\eta]=[} & X, Y]+L_{X} \eta-i_{Y} d \xi+i_{Y} i_{X} H \\
& -F(X, Y)+i_{X} d t-i_{Y} d r \\
& +2 c(t d r)+2 c\left(i_{X} F t\right)-2 c\left(i_{Y} F r\right) .
\end{aligned}
$$

It can be checked from the Bianchi identity (4.12) that $(E,\langle\cdot, \cdot\rangle,[\cdot, \cdot], \pi)$ satisfies the axioms of a Courant algebroid (see [61, Sec. 2.3.3]).

Definition 4.1. A Courant algebroid $(E,\langle\cdot, \cdot\rangle,[\cdot, \cdot], \pi)$ over a manifold $M$ consists of a vector bundle $E \rightarrow M$ together with a non-degenerate symmetric bilinear form $\langle\cdot, \cdot\rangle$ on $E$, a (Dorfman) bracket $[\cdot, \cdot]$ on the sections $\Omega^{0}(E)$, and a bundle map $\pi: E \rightarrow T M$ such that the following properties are satisfied, for $e, e^{\prime}, e^{\prime \prime} \in \Omega^{0}(E)$ and $\phi \in C^{\infty}(M)$ :

(D1): $\left[e,\left[e^{\prime}, e^{\prime \prime}\right]\right]=\left[\left[e, e^{\prime}\right], e^{\prime \prime}\right]+\left[e^{\prime},\left[e, e^{\prime \prime}\right]\right]$,

(D2): $\pi\left(\left[e, e^{\prime}\right]\right)=\left[\pi(e), \pi\left(e^{\prime}\right)\right]$

(D3): $\left[e, \phi e^{\prime}\right]=\pi(e)(\phi) e^{\prime}+\phi\left[e, e^{\prime}\right]$,

(D4): $\pi(e)\left\langle e^{\prime}, e^{\prime \prime}\right\rangle=\left\langle\left[e, e^{\prime}\right], e^{\prime \prime}\right\rangle+\left\langle e^{\prime},\left[e, e^{\prime \prime}\right]\right\rangle$,

(D5): $\left[e, e^{\prime}\right]+\left[e^{\prime}, e\right]=2 \pi^{*} d\left\langle e, e^{\prime}\right\rangle$.

Automorphisms of this object were characterized in [61, Prop. 2.23]. For our discussion, we just need the infinitesimal automorphisms, given by a linear subspace

$$
\text { Lie Aut } E \subset \Omega^{0}(T) \oplus \Omega^{1}(i \mathbb{R}) \oplus \Omega^{2} \text {. }
$$

Proposition $4.2([61])$. The Lie algebra of infinitesimal automorphisms of $E$ is given by

$$
\text { Lie Aut } E=\left\{(V, a, B): \mathcal{L}_{V} F=d a, \mathcal{L}_{V} H=-d B+2 c(a \wedge F)\right\} .
$$

From (4.14) and (4.7), we obtain a natural map

$$
\text { Lie Aut } E \rightarrow \text { Lie Aut } E:(V, r, B) \mapsto\left(V, d r+i_{V} F, B\right) \text {. }
$$

In fact, the image defines a subspace closed under the Lie bracket (see Corollary 4.2), which justifies the notation and gives the desired interpretation of 
Lie Aut $E$. The space of sections $\Omega^{0}(E)$ corresponds to the inner symmetries of $E$, via the natural action induced by the Dorfman bracket.

To interpret geometrically the space of parameters $A^{1} \oplus \Omega^{2}$ in (4.8) and (4.9), we need generalized metrics. A generalized metric on $E$ is given by a linear subspace

$$
V_{+} \subset E
$$

such that the restriction of the pairing on $E$ is non-degenerate. For simplicity, we assume $c<0$ and the induced metric on $V_{+}$to be positive definite. Then, a generalized metric is equivalent to a Riemannian metric $g$ on $M$ and an isotropic splitting of $E$ that, using the canonical isotropic splitting of (4.11), can be regarded as an orthogonal transformation by $(b, a) \in \Omega^{2} \oplus \Omega^{1}(\operatorname{ad} P)$. Hence, the fixed solution of the abelian system (2.3) determines a generalized metric $V_{+}$, such that the metric is determined by the $\mathrm{SU}(3)$-structure $(\Omega, \omega)$, $b=0$ and $a=0$. Variations of $V_{+}$are given by variations of the metric, the imaginary 1-form $a$ and the 2-form $b \in \Omega^{2}$, which are precisely the elements parameterized by $A^{1} \oplus \Omega^{2}$.

The conclusion is that the finite-dimensional vector space $H^{1}\left(\AA^{*}\right)$ corresponds to a space of infinitesimal variations of $V_{+}$as a generalized metric modulo the natural symmetries of the smooth Courant algebroid $E$ (4.11), while $H^{1}\left(\widehat{A}^{*}\right)$ is cut out by inner symmetries of $E$. Of course, $H^{1}\left(\AA^{*}\right)$ and $H^{1}\left(\widehat{A}^{*}\right)$ contain more information related to variations of the $\mathrm{SU}(3)$-structure on $M$ and the abelian system itself, that we ignore at this point of our discussion.

4.5. Relation with generalized geometry: symmetries. We address now the relation between generalized geometry and the space of infinitesimal variations of the Strominger system $H^{1}\left(S^{*}\right)$, constructed in Section 3 , We start our discussion with the definition of a suitable transitive Courant algebroid over $M$ and the characterization of its group of automorphisms. We follow the notation introduced in Section 3.1.

We fix a solution $(\Omega, A, \nabla, \omega)$ of the Strominger system (1.1). We define the quantities $H:=d^{c} \omega$ and

$$
F=F_{A}+R_{\nabla}
$$

the curvature of $\theta=\nabla \times A$, and consider the covariant derivative $d^{\theta}$ induced by $\theta$ in ad $P$. With this notation, the Bianchi identity (last equation in (1.1)) can be written as

$$
d H=c(F \wedge F) .
$$

Consider the vector bundle

$$
E=T \oplus \operatorname{ad} P \oplus T^{*}
$$

endowed with the symmetric pairing

$$
\langle X+r+\xi, Y+t+\eta\rangle=\frac{1}{2}(\eta(X)+\xi(Y))+c(r, t),
$$

and the canonical projection

$$
\pi: E \rightarrow T
$$


Using the quantities $H, \theta$ and $F$, we can endow $\Omega^{0}(E)$ with a Dorfman bracket

$$
\begin{aligned}
{[X+r+\xi, Y+t+\eta]=[} & X, Y]+\mathcal{L}_{X} \eta-i_{Y} d \xi+i_{Y} i_{X} H \\
& -[r, t]-F(X, Y)+d_{X}^{\theta} t-d_{Y}^{\theta} r \\
& +2 c\left(d^{\theta} r, t\right)+2 c(F(X, \cdot), t)-2 c(F(Y, \cdot), r) .
\end{aligned}
$$

Following [13], it can be checked from the Bianchi identity (4.15) that the tuple $(E,\langle\cdot, \cdot\rangle,[\cdot, \cdot], \pi)$ satisfies the axioms of a Courant algebroid (see Definition 4.1). An indirect way of proving that the previous axioms are satisfied is to construct the Courant algebroid from reduction [10] (see [30, Section 2] and [6]). We will use the fact that $E$ is obtained from reduction of an exact Courant algebroid over $P$ in Corollary 4.2 .

Let Aut $E$ denote the space of automorphisms of the vector bundle $E$ that preserve the bracket and the pairing. Given $f \in$ Aut $E$ we denote by $\check{f} \in$ Diff the diffeomorphism on $M$ that it covers. The property (D3) gives $\pi \circ f=d \check{f} \circ \pi$, so $f$ is compatible with the anchor map. Note that any $\nu \in \mathrm{O}(\operatorname{ad} P)$ covering a diffeomorphism $\check{f}$ defines an orthogonal transformation of $E \cong T+\operatorname{ad} P+T^{*}$ given by

$$
f_{\nu}:=\left(\begin{array}{ccc}
d \check{f} & & \\
& \nu & \\
& & \left(d \check{f}^{*}\right)^{-1}
\end{array}\right) .
$$

Orthogonal transformations of $E$ compatible with $\pi$ and acting as the identity on ad $P$, and hence covering the identity, are of the form

$$
(B, a):=\left(\begin{array}{ccc}
\mathrm{Id} & 0 & 0 \\
a & \mathrm{Id} & 0 \\
B-c(a, a) & -2 c(a, \cdot) & \mathrm{Id}
\end{array}\right),
$$

with $B \in \Omega^{2}, a \in \Omega^{1}($ ad $P)$. To characterize the group $\operatorname{Aut}(E)$, we note that an element $\nu \in O(\operatorname{ad} P)$ covering $\check{f} \in$ Diff acts on the covariant derivative $d^{\theta}$ by

$$
i_{V}\left(\nu \cdot d^{\theta}\right) r=\nu \cdot\left(i_{\check{f}^{*} V} d^{\theta}\left(\nu^{-1} \cdot r\right)\right),
$$

where we use the action of $\nu$ on a section $r \in \Omega^{0}(\operatorname{ad} P)$, given by $\nu \cdot r(x)=$ $\nu\left(r_{\check{f}^{-1}(x)}\right)$, for $x \in M$. Similarly, we have an action on $F$, given by

$$
\left(i_{W} i_{V}(\nu \cdot F)\right)(x)=\nu\left(i_{\check{f}^{*} W} i_{\check{f}^{*} V} F\left(\check{f}^{-1}(x)\right)\right) .
$$

Furthermore, any $a \in \Omega^{1}(\operatorname{ad} P)$ can be used to deform the connection $\theta$ to a new connection $\theta+a: T P \rightarrow V P$ on $P$ such that

$$
i_{V} d^{\theta+a} r=\left[\theta^{\perp} V-i_{V} a, r\right]=i_{V}\left(d^{\theta} r+[a, r]\right),
$$

where the bracket in the middle expression denotes Lie bracket of vector fields on $P$, while the bracket in the right expression denotes the bracket on ad $P$. The induced curvature is (note that $d^{\theta} \circ d^{\theta}=[F, \cdot]$ )

$$
F_{\theta+a}=F+d^{\theta} a+\frac{1}{2}[a, a],
$$

where $[a, a](V, W)=2[a(V), a(W)]$. 
Proposition 4.3. The group $\operatorname{Aut}(E)$ is the set of orthogonal transformations

$$
\begin{aligned}
\left\{f_{\nu}(B, a) \mid\right. & \nu \in \operatorname{Aut}(\operatorname{ad} P,\langle\cdot, \cdot\rangle,[\cdot, \cdot]), B \in \Omega^{2}, a \in \Omega^{1}(\operatorname{ad} P), \\
& d^{\theta}=\nu \cdot d^{\theta+a}, \quad F=\nu \cdot F_{\theta+a}, \\
& \left.\check{f}^{*} H=H-d B+2 c(a, F)+c\left(a \wedge d^{\theta} a\right)+\frac{1}{3} c(a \wedge[a, a])\right\}
\end{aligned}
$$

together with the product given, for $f=f_{\nu}(B, a)$ and $f^{\prime}=f_{\nu^{\prime}}\left(B^{\prime}, a^{\prime}\right)$, by

$$
f \circ f^{\prime}=f_{\nu \nu^{\prime}}\left(\check{f}^{\prime *} B+B^{\prime}+c\left(\left(\nu^{\prime-1} \cdot a\right) \wedge a^{\prime}\right), \nu^{\prime-1} \cdot a+a^{\prime}\right) .
$$

Proof. Given $f \in \operatorname{Aut}(E)$, the compatibility with the anchor map gives that the first row is $(d \check{f}, 0,0)$. Then, as $f$ preserves the pairing, we have that the entry $(2,3)$ vanishes and $f$ is of the form

$$
f=\left(\begin{array}{ccc}
d \check{f} & 0 & 0 \\
* & \nu & 0 \\
* & * & d \check{f}^{-1}
\end{array}\right),
$$

for some $\nu \in \mathrm{O}(\operatorname{ad} P)$. The transformation $f_{\nu}^{-1} f$ preserves the pairing, is the identity on ad $P$ and is compatible with $\pi$, so it has to be $(B, a)$ for some $B \in \Omega^{2}$ and $a \in \Omega^{1}(\operatorname{ad} P)$. Thus, $f=f_{\nu}(B, a)$. We next check what are the constraints on $\nu, B, a$ for $f$ to preserve the Courant bracket.

First, $\nu$ must preserve the bracket on ad $P$. If that condition is satisfied,

$$
f_{\nu}\left[f_{\nu}^{-1} \cdot, f_{\nu}^{-1} \cdot\right]_{d^{\theta}, F, H}=[\cdot, \cdot]_{\nu \cdot d^{\theta}, \nu \cdot F, \check{f}_{*} H} \cdot
$$

On the other hand, after several elementary calculations, we have

$$
(B, a)[(-B,-a) \cdot,(-B,-a) \cdot]_{d^{\theta}, F, H}=[\cdot, \cdot]_{d^{\theta+a}, F_{\theta+a}, H^{\prime}}
$$

with

$$
H^{\prime}=H-d B+2 c(a, F)+c\left(a, d^{\theta} a\right)+\frac{1}{3} c(a,[a, a]),
$$

which give the conditions for $\operatorname{Aut}(P)$.

For the composition law, note that for $\nu^{\prime} \in \mathrm{O}(\operatorname{ad} P)$,

$$
(B, a) \nu_{*}^{\prime}=\nu_{*}^{\prime}\left(\check{g}^{*} B,\left(\nu^{\prime}\right)^{-1} \check{g}^{*} a\right)
$$

and that $(B, a)\left(B^{\prime}, a^{\prime}\right)=\left(B+B^{\prime}+c\left(a \wedge a^{\prime}\right), a+a^{\prime}\right)$.

Remark 4.2. The previous result applies in the more general setup studied in [13, for an arbitrary transitive Courant algebroid not necessarily obtained from reduction. Note that, for pure $a$-field transformations, the condition $d^{\theta}=d^{\theta+a}$ is in general very strong, as it forces $a$ to take values in the centre of the bundle of Lie algebras.

For the next result, we use the fact that $E$ is obtained from reduction of an exact Courant algebroid over $P$.

Corollary 4.1. The group Aut $E$ fits into an exact sequence of groups

$$
0 \longrightarrow \Omega_{c l}^{2} \longrightarrow \text { Aut } E \stackrel{q}{\longrightarrow} \text { Aut } T P / G
$$

where $\Omega_{c l}^{2}$ denotes the space of closed 2 -forms on $M$ and Aut $T P / G$ is the group of automorphisms of the Lie algebroid $T P / G$. 
Proof. When projecting to $T P / G \cong T \oplus$ ad $P$, the Courant bracket on sections of $E$ corresponds to the Lie bracket on sections of $T P / G$. From Proposition 4.3, we see that $q^{-1}(\mathrm{Id})$ consists of the elements such that $\nu=\mathrm{Id}, \check{f}=\mathrm{Id}$, $a=0$, that is, of the exponentials of 2 -forms $B$, which must be closed by the last defining condition of $\operatorname{Aut}(E)$.

The group Aut $P$ can be regarded as a subgroup of $\operatorname{Aut}(T P / G)$. Given $g \in$ Aut $P$ covering a diffeomorphism $\check{g}$, the corresponding element $d g \in$ $\operatorname{Aut}(T P / G)$ preserves the vertical part, i.e. ad $P$, and hence defines an element

$$
\nu^{g}:=d g_{\mid \text {ad } P} \in \operatorname{Aut}(\operatorname{ad} P,\langle\cdot, \cdot\rangle,[\cdot, \cdot]) .
$$

The element $d g \in \operatorname{Aut}(T P / G)$ is described, in terms of the splitting $T \oplus \operatorname{ad} P$, by

$$
d g=\left(\begin{array}{cc}
d \check{g} & \\
& \nu^{g}
\end{array}\right)\left(\begin{array}{cc}
1 & \\
g^{-1} \cdot \theta-\theta & 1
\end{array}\right),
$$

where the isomorphism $T P / G \cong T \oplus$ ad $P$ is given by a connection $\theta$. We denote by $f_{g}=f_{\nu^{g}}$ the induced orthogonal transformation of $E$. The next result is a direct consequence of Proposition 4.3 .

Corollary 4.2. Define Aut $E=q^{-1}($ Aut $P)$. Then, there is an exact sequence of groups

$$
0 \rightarrow \Omega_{c l}^{2} \rightarrow \text { Aut } E \rightarrow \text { Aut } P .
$$

Elements in Aut $E$ correspond to transformations $f_{g}\left(B, a^{g}\right)$, with $g \in$ Aut $P$ covering $\check{g}, B \in \Omega^{2}$ and $a^{g}:=g^{-1} \cdot \theta-\theta$, which satisfy

$$
\check{g}^{*} H=H-d B+2 c\left(a^{g}, F\right)+c\left(a^{g}, d^{\theta} a^{g}\right)+\frac{1}{3} c\left(a^{g},\left[a^{g}, a^{g}\right]\right) .
$$

Regarding Aut $E \subset$ Aut $P \times \Omega^{2}$, the group structure is given by

$$
(g, B)\left(g^{\prime}, B^{\prime}\right)=\left(g g^{\prime}, \check{g}^{\prime *} B+B^{\prime}+c\left(\left(g^{\prime-1} \cdot a^{g}\right) \wedge a^{g^{\prime}}\right)\right),
$$

with Lie algebra Lie Aut $E \subset$ Lie Aut $P \oplus \Omega^{2}$ given by

$$
\text { Lie Aut } E=\left\{\hat{V}+B: \mathcal{L}_{V} H=-d B+2 c((\hat{V} \cdot \theta) \wedge F)\right\}
$$

where $\hat{V} \cdot \theta$ denotes the infinitesimal action of $\hat{V} \in$ Lie Aut $P$ on $\theta$ and $V \in$ $\Omega^{0}(T)$ is the vector on $M$ covered by $\hat{V}$.

Proof. The proof follows from Proposition 4.3, noticing that $g \cdot\left(\theta+a^{g}\right)=\theta$. The claim about the Lie algebra follow from taking one-parameter subgroups of generalized diffeomorphisms in Aut $E$.

Remark 4.3. Condition (4.22) is better understood in the total space of $P$, in terms of the transgression formula for the Chern-Simons 3-form for the connection $\theta$ (see [30]). For this, we note that given $g \in$ Aut $P$, the elements in $q^{-1}(g)$ correspond to $B \in \Omega^{2}$ such that

$$
d\left(B+c\left(g^{-1} \theta \wedge \theta\right)\right)=H-C S(\theta)-g^{*}(H-C S(\theta)) .
$$


4.6. The complex $\stackrel{\circ}{S}^{*}$ and the elliptic complex $\widehat{S}^{*}$. We use next the symmetries of the Courant algebroid $E$ to construct an exact diagram of linear maps

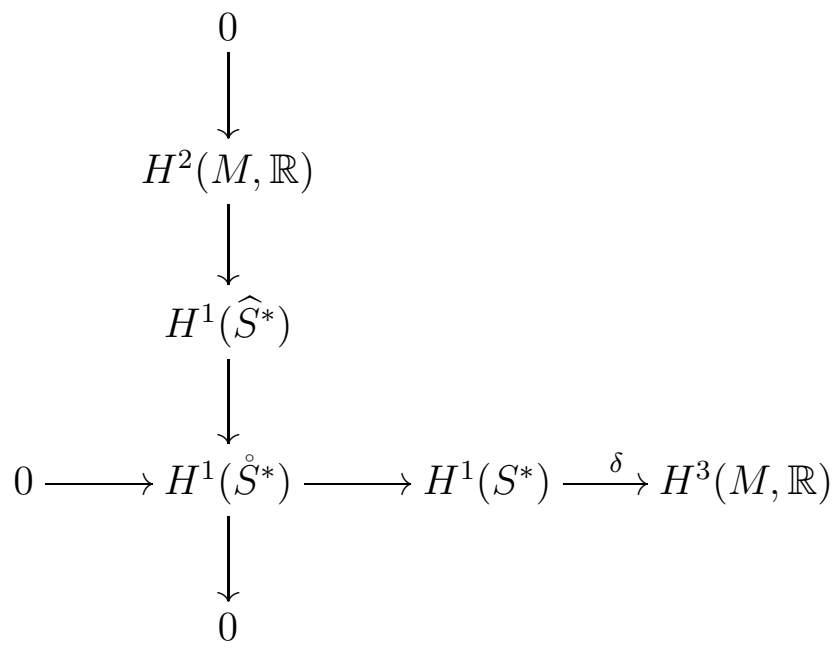

such that $H^{1}\left(\stackrel{\circ}{S}^{*}\right)$ and $H^{1}\left(\widehat{S}^{*}\right)$ have a natural interpretation in generalized geometry. To make the link with the construction in Section 3, we need to consider the reduction

$$
P_{h} \subset P
$$

of the bundle $P$ to $K \times \mathrm{U}(3)$ provided by the hermitian structure $(\Omega, \omega)$ of the fixed solution. We define Lie Aut $E_{h} \subset$ Lie Aut $E$ by

$$
\text { Lie Aut } E_{h}=\left\{(\hat{V}, B) \in \text { Lie Aut } E: \theta \hat{V}-\nabla V \in \Omega^{0}\left(\operatorname{ad} P_{h}\right)\right\}
$$

where $\theta \hat{V}$ denotes the vertical part of $\hat{V}$ with respect to the connection $\theta$ and $V \in \Omega^{0}(T)$ is the vector field covered by $\hat{V}$.

Let $H^{1}\left(S^{*}\right)$ be the finite-dimensional vector space of infinitesimal variations of the given solution (see Section 3). Then, by (3.13) there is a natural linear map

given by

$$
\delta: H^{1}\left(S^{*}\right) \rightarrow H^{3}(M, \mathbb{R}),
$$

$$
[(\dot{\Omega}, \dot{\theta}, \dot{\omega})] \mapsto\left[J d \dot{\omega}-J(d \omega)^{\dot{J} J}-2 c(\dot{\theta} \wedge F)\right] .
$$

We define a sequence

$$
S^{0} \oplus \Omega^{2} \stackrel{\stackrel{\circ}{\mathbf{P}}}{\longrightarrow} S^{1} \oplus \Omega^{2} \stackrel{\stackrel{\circ}{\mathbf{L}}}{\longrightarrow} \stackrel{\circ}{\mathcal{R}}
$$

where we follow the notation of Section 3 , Here,

$$
\dot{\mathcal{R}}:=\Omega^{3,1} \oplus \Omega^{2,2} \oplus \Omega^{0,2}\left(\operatorname{ad} P_{h}\right) \oplus \Omega^{3} \oplus \Omega^{5} \oplus \Omega^{6}\left(\operatorname{ad} P_{h}\right)
$$

and the maps $\stackrel{\circ}{\mathbf{P}}$ and $\stackrel{\circ}{\mathbf{L}}$ are defined by

$$
\stackrel{\circ}{\mathbf{P}}(V, r, B)=(\mathbf{P}(V, r),-B)
$$

and

where

$$
\stackrel{\circ}{\mathbf{L}}=\mathbf{L}_{1} \oplus \mathbf{L}_{2} \oplus \stackrel{\circ}{\mathbf{L}}_{3} \oplus \mathbf{L}_{4} \oplus \mathbf{L}_{5},
$$

$$
\stackrel{\circ}{\mathbf{L}}_{3}(\dot{\Omega}, \dot{\theta}, \dot{\omega}, b)=J d \dot{\omega}-J(d \omega)^{\dot{J} J}-2 c(\dot{\theta} \wedge F)-d b .
$$


For the next result, we note that we can regard Lie Aut $E_{h} \subset S^{0} \oplus \Omega^{2}$ by using the natural map

$$
(\hat{V}, B) \rightarrow(V, \theta \hat{V}-\nabla V, B)
$$

which satisfies

$$
\mathbf{P}(V, \theta \hat{V}-\nabla V)=\left(\mathcal{L}_{V} \Omega, \hat{V} \cdot \theta, \mathcal{L}_{V} \omega\right) .
$$

Lemma 4.3. The sequence (4.24) induces a complex

$$
\text { Lie Aui } E_{h} \stackrel{\stackrel{\circ}{\longrightarrow}}{\longrightarrow} S^{1} \oplus \Omega^{2} \stackrel{\stackrel{\circ}{\longrightarrow}}{\longrightarrow} \stackrel{\circ}{\mathcal{R}}
$$

by restriction of the first arrow, whose cohomology $H^{1}\left(\stackrel{\circ}{S}^{*}\right)$ is naturally isomorphic to the kernel of (4.23). Consequently, the cohomology of $\stackrel{\circ}{S}^{*}$ is finite dimensional.

The proof follows as in Lemma 4.2. Consider now the vector bundle

$$
E_{h}=T \oplus \operatorname{ad} P_{h} \oplus T^{*}
$$

and note that $\Omega^{0}\left(E_{h}\right)=S^{0} \oplus \Omega^{1}$. We define a sequence of differential operators

$$
\left(\widehat{S}^{*}\right) \quad \quad \Omega^{0}\left(E_{h}\right) \stackrel{\stackrel{\circ}{\longrightarrow}}{\longrightarrow} S^{1} \oplus \Omega^{2} \stackrel{\stackrel{\circ}{\mathbf{L}}}{\longrightarrow} \mathcal{R},
$$

where

$$
\stackrel{\circ}{\mathbf{P}}(V, r, \xi)=\left(\mathbf{P}(V, r), d \xi+i_{V}\left(d^{c} \omega\right)-2 c(r+\nabla V, F)\right) .
$$

A straightforward calculation shows that

$$
\left(V, r,-d \xi-i_{V}\left(d^{c} \omega\right)+2 c(r+\nabla V, F)\right) \in \text { Lie Aut } E_{h}
$$

and therefore (4.26) is a complex of differential operators.

Proposition 4.4. The sequence (4.26) is an elliptic complex of differential operators of degree 1 . There is an exact sequence

$$
0 \rightarrow H^{2}(M, \mathbb{R}) \rightarrow H^{1}\left(\widehat{S}^{*}\right) \rightarrow H^{1}\left(\stackrel{\circ}{S}^{*}\right) \rightarrow 0
$$

where $H^{1}\left(\widehat{S}^{*}\right)$ denotes the cohomology of (4.26).

The proof is analogue to the proof of Proposition 4.1.

Remark 4.4. The ellipticity of the complex (4.26) gives an alternative proof of the finite-dimensionality of $H^{1}\left(\stackrel{\circ}{S}^{*}\right)$.

4.7. Relation with generalized geometry: metrics. We finish this section by providing an interpretation of $S^{1} \oplus \Omega^{2}$ — the middle step in the complexes (4.25) and (4.26) - as a space of infinitesimal variations of a generalized metric on $E$. For this, we recall a few basic facts about generalized metrics on the Courant algebroid $E$ following 30 . This will provide the necessary background for Section 5 .

Let $(t, s)$ be the signature of the pairing on the Courant algebroid $E$. A generalized metric of signature $(p, q)$, or simply a metric on $E$, is a reduction of the $O(t, s)$-bundle of frames of $E$ to

$$
O(p, q) \times O(t-p, s-q) \subset O(t, s) .
$$


Alternatively, it is given by a subbundle

$$
V_{+} \subset E
$$

such that the restriction of the metric on $E$ to $V_{+}$is a non-degenerate metric of signature $(p, q)$. We denote by $V_{-}$the orthogonal complement of $V_{+}$on $E$. A generalized metric determines a vector bundle isomorphism

$$
G: E \rightarrow E,
$$

with \pm 1 -eigenspace $V_{ \pm}$, which is symmetric, $G^{*}=G$, and squares to the identity, $G^{2}=\mathrm{Id}$. The endomorphism $G$ determines completely the metric, as $V_{+}$ is recovered by

$$
V_{+}=\operatorname{Ker}(G-\mathrm{Id}) .
$$

Definition $4.2([30])$. A metric $V_{+}$of arbitrary signature is admissible if

$$
V_{+} \cap T^{*}=\{0\} \quad \text { and } \quad \operatorname{rk} V_{+}=\operatorname{rk} E-\operatorname{dim} M .
$$

We recall the basic result that we need in order to understand the parameters encoded by an admissible generalized metric.

Proposition 4.5 ([30]). An admissible metric $V_{+}$on $E$ is equivalent to a pair given by a metric $g$ on $M$ and an isotropic splitting. Using the canonical isotropic splitting of (4.16), $V_{+}$is given by a metric $g$ on $M$ and an orthogonal transformation by $(b, a) \in \Omega^{2} \oplus \Omega^{1}($ ad $P)$

$$
V_{+}=(-b,-a)\{X+g(X)+r: X \in T, r \in \operatorname{ad} P\} .
$$

An admissible metric determines a connection $\theta^{\prime}=\theta+a$ on $P$ and a 3-form $H^{\prime}$ on $M$ given by (4.18), such that the bracket in the splitting provided by $V_{+}$ is given by (4.17).

From the previous result, we note that the fixed solution of the Strominger system determines an admissible generalized metric $V_{+}$, given by the metric determined by the $\mathrm{SU}(3)$-structure $(\Omega, \omega)$ and the canonical isotropic splitting, with connection $\theta$ and 3 -form $H=d^{c} \omega$. Furthermore, cocycles for $S^{*}$ in $S^{1} \oplus \Omega^{2}$ correspond to infinitesimal variations of the admissible metric $V_{+}$.

Therefore, the finite-dimensional vector space $H^{1}\left(\stackrel{S}{S}^{*}\right)$ corresponds to a space of infinitesimal variations of $V_{+}$as a generalized metric modulo natural symmetries of the smooth Courant algebroid $E$ (4.16), while $H^{1}\left(\widehat{S}^{*}\right)$ is cut out by inner symmetries of $E$.

\section{Killing SPINORS IN GENERALIZED GEOMETRY}

In this section we introduce a natural notion of Killing spinor in generalized geometry. The Killing spinor equations

$$
D_{+}^{\phi} \eta=0, \quad \not D_{-}^{\phi} \eta=0,
$$

depend on a generalized metric $V_{+}$on a smooth Courant algebroid $E$ over $M$ and a smooth function $\phi \in C^{\infty}(M)$. We specify to the case of a spin manifold of dimension six and study the equations (5.1) in two different cases. Firstly, when $E$ is exact, we show that a solution of (5.1) is equivalent to a metric on $M$ with holonomy SU(3). Secondly, for a suitable choice of transitive Courant 
algebroid $E$, by applying the original result of Strominger and Hull [39, 63], we prove that the Killing spinor equations (5.1) correspond to the Strominger system.

The present section gives a precise interpretation of the vector spaces $H^{1}\left(\stackrel{\circ}{S}^{*}\right)$ and $H^{1}\left(\widehat{S}^{*}\right)$ constructed in Section 4, as spaces of infinitesimal deformations of solutions of the Killing spinor equations (5.1) modulo infinitesimal symmetries of a Courant algebroid, offering a conceptual explanation of the appearance of generalized geometry in the study of the Strominger system. In addition, it provides a unifying framework for the theory of the Strominger system and the well-established theory of metrics with holonomy $\mathrm{SU}(3)$, which we expect will have future applications in the former.

5.1. The canonical Levi-Civita connection. Let $E$ be a smooth, transitive, Courant algebroid over a smooth manifold $M$ of arbitrary dimension, that is, a vector bundle satisfying the axioms of Definition 4.1 and such that the anchor map

$$
\pi: E \rightarrow T
$$

is surjective. In this section we introduce a canonical notion of Levi-Civita connection on $E$ - a natural torsion-free connection associated to an admissible generalized metric. For simplicity, we will assume that $E$ is obtained from reduction of an exact Courant algebroid on a principal $G$-bundle $P$, as in [30, Sec. 2]. The general case follows easily from [13.

Recall that a generalized connection $D$ (or simply, a connection) on $E$ is a first order differential operator

$$
D: \Omega^{0}(E) \rightarrow \Omega^{0}\left(E^{*} \otimes E\right)
$$

satisfying the Leibniz rule $D_{e}\left(\phi e^{\prime}\right)=\phi D_{e} e^{\prime}+\pi(e)(\phi) e^{\prime}$, for $e, e^{\prime} \in \Omega^{0}(E)$ and $\phi \in C^{\infty}(M)$. We will only consider connections compatible with the inner product on $E$, that is, satisfying

$$
\pi(e)\left(\left\langle e^{\prime}, e^{\prime \prime}\right\rangle\right)=\left\langle D_{e} e^{\prime}, e^{\prime \prime}\right\rangle+\left\langle e^{\prime}, D_{e} e^{\prime \prime}\right\rangle .
$$

The space of connections on $E$ is an affine space modelled on $\Omega^{0}\left(E^{*} \otimes \mathfrak{o}(E)\right)$.

Following [36], we first introduce the notion of Gualtieri-Bismut connection. Given an admissible metric $V_{+}$(see Definition 4.2), we define

$$
C^{+}=(\operatorname{ad} P)^{\perp} \subset V_{+} .
$$

We can associate an endomorphism of the vector bundle $E$ such that $C\left(V_{+}\right)=$ $V_{-}$and $C\left(V_{-}\right)=C_{+}$, defined by

$$
C=\pi_{\mid V_{-}}^{-1} \circ \pi \circ \Pi_{+}+\pi_{\mid C_{+}}^{-1} \circ \pi \circ \Pi_{-}
$$

where

$$
\Pi_{ \pm}=\frac{1}{2}(\operatorname{Id} \pm G): E \rightarrow V_{ \pm}
$$

denote the orthogonal projections. In the canonical splitting provided by $V_{+}$

$$
E \cong T \oplus \operatorname{ad} P \oplus T^{*}
$$

we have (see Proposition 4.5)

$$
V_{+}=\{X+g(X)+r: X \in T, r \in \operatorname{ad} P\}
$$


and then we can write explicitly

$$
C(X+r+g X)=X-g X \quad \text { and } \quad C(Y-g Y)=Y+g Y .
$$

Definition 5.1. The Gualtieri-Bismut connection $D^{B}$ of $V_{+}$on $E$ is defined by

$$
D_{e}^{B} e^{\prime}=\left[e_{-}, e_{+}^{\prime}\right]_{+}+\left[e_{+}, e_{-}^{\prime}\right]_{-}+\left[C e_{-}, e_{-}^{\prime}\right]_{-}+\left[C e_{+}, e_{+}^{\prime}\right]_{+},
$$

where $e_{ \pm}=\Pi_{ \pm} e$.

We check now that $D^{B}$ is well-defined.

Proposition 5.1. The expression (5.4) defines a well-defined connection on $E$ compatible with $V_{+}$, that is, $D^{B}$ sends $V_{ \pm}$to $V_{ \pm}$.

Proof. The Leibniz rule and the facts that $D^{B}$ preserves $V_{ \pm}$and the inner product on $E$ follow exactly as in [36, Th. 3.1]. To prove that $D_{\phi e}^{B} e^{\prime}=\phi D_{e}^{B} e^{\prime}$ for any $\phi \in C^{\infty}(M)$, using the properties of the Dorfman bracket we calculate

$$
\begin{aligned}
D_{f e_{-}}^{B} e^{\prime}= & {\left[f e_{-}, e_{+}^{\prime}\right]_{+}+\left[C\left(f e_{-}\right), e_{-}^{\prime}\right]_{-} } \\
= & -f\left[e_{+}^{\prime}, e_{-}\right]_{+}-\pi\left(e_{+}^{\prime}\right)(f)\left(e_{-}\right)_{+} \\
& -f\left[e_{-}^{\prime}, C e_{-}\right]_{-}-\pi\left(e_{-}^{\prime}\right)(f)\left(C e_{-}\right)_{-} \\
= & f\left(\left[e_{-}, e_{+}^{\prime}\right]_{+}+\left[C e_{-}, e_{-}^{\prime}\right]_{-}\right)
\end{aligned}
$$

and similarly for $D_{f e_{+}}^{B} e^{\prime}$.

The generalized torsion [36] of a connection $D$ on $E$ is a totally skew tensor $T_{D} \in \Lambda^{3} E^{*}$ defined by

$$
T_{D}(a, b, c)=\left\langle D_{a} b-D_{b} a-[[a, b]], c\right\rangle+\frac{1}{2}\left(\left\langle D_{c} a, b\right\rangle-\left\langle D_{c} b, a\right\rangle\right),
$$

where

$$
[[a, b]]=\frac{1}{2}([a, b]-[b, a])
$$

is the skew-symmetrization of the Dorfman bracket on sections of $E$. A connection with vanishing torsion will be referred as a torsion-free connection. By analogy with hermitian geometry, we introduce the following notion of LeviCivita connection associated to a generalized metric.

Definition 5.2. The canonical Levi-Civita connection of $V_{+}$is defined by

$$
D^{L C}=D^{B}-\frac{1}{3} T_{D^{B}}
$$

where we identify the torsion $T_{D^{B}}$ with the element $\langle\cdot, \cdot\rangle^{-1} T_{D^{B}} \in E \otimes \Lambda^{2} E^{*}$.

By construction, $D^{L C}$ is a natural object on $E$, that is, given an automorphism $f \in$ Aut $E$ and a generalized metric $V_{+}$, we have

$$
f_{*}\left(D^{L C}\left(V_{+}\right)\right)=D^{L C}\left(f\left(V_{+}\right)\right) .
$$

As in 30 , we can modify $D^{L C}$ by elements in $E^{*}$, while preserving the torsionfree property - hence, torsion-free, metric connections are not unique (see also [14]). Note that any other compatible generalized connection differs from $D^{L C}$ by

$$
\chi \in E^{*} \otimes\left(\mathfrak{o}\left(V_{+}\right) \oplus \mathfrak{o}\left(V_{-}\right)\right) .
$$


Given $\varphi \in E^{*}$, consider the Weyl term

$$
\chi^{\varphi} \in E^{*} \otimes \mathfrak{o}(E)
$$

defined by

$$
\chi_{e}^{\varphi} e^{\prime}=\varphi\left(e^{\prime}\right) e-\left\langle e, e^{\prime}\right\rangle\langle\cdot, \cdot\rangle^{-1} \varphi
$$

We introduce the notation

$$
\chi_{e}^{ \pm \pm \pm} e^{\prime}=\Pi_{ \pm} \chi_{e_{ \pm}}\left(e_{ \pm}^{\prime}\right)
$$

for $\chi \in E^{*} \otimes(\mathfrak{o}(E))$ and $e, e^{\prime} \in E$. Then, we obtain a new torsion-free, compatible, generalized connection by the formula

$$
D^{\varphi}=D^{L C}+\frac{1}{3\left(\operatorname{rk} V_{+}-1\right)}\left(\chi^{\varphi}\right)^{+++}+\frac{1}{3\left(\operatorname{rk} V_{-}-1\right)}\left(\chi^{\varphi}\right)^{---} .
$$

Once more, we have that the connection $D^{\varphi}$ is natural, in the following sense

$$
f_{*}\left(D^{f^{*} \varphi}\left(V_{+}\right)\right)=D^{\varphi}\left(f\left(V_{+}\right)\right),
$$

for any $f \in$ Aut $E$.

Remark 5.1. A more natural approach to the previous construction would be to derive $D^{\varphi}$ as the canonical Levi-Civita connection on a modified version of a Courant algebroid, which keeps track of conformal changes in the pairing $\langle\cdot, \cdot\rangle$.

5.2. Some explicit formulae. We fix a generalized metric $V_{+}$on $E$ and consider the associated splitting (5.2) and triple $(g, H, \theta)$ (see Proposition 4.5). We denote by $c$ the symmetric pairing induced by $\langle\cdot, \cdot\rangle$ in ad $P$. We define connections on $T$ with skew torsion, compatible with the metric $g$, given by

$$
\begin{aligned}
\nabla^{ \pm} & =\nabla^{g} \pm \frac{1}{2} g^{-1} H, \\
\nabla^{ \pm 1 / 3} & =\nabla^{g} \pm \frac{1}{6} g^{-1} H,
\end{aligned}
$$

where $\nabla^{g}$ denotes the Levi-Civita connection of the metric $g$ on $M$. Consider the covariant derivative $d^{\theta}$ on ad $P$ determined by $\theta$. Considering

$$
\begin{aligned}
& a_{+}=X+r+g X, \\
& b_{-}=Y-g Y, \\
& c_{+}=Z+t+g Z, \\
& d_{-}=W-g W,
\end{aligned}
$$

we have

$$
\begin{aligned}
& D_{a_{+}}^{B} c_{+}=2 \Pi_{+}\left(\nabla_{X}^{+} Z+g^{-1} c\left(i_{X} F, t\right)\right)+d_{X}^{\theta} t-F(X, Z), \\
& D_{b_{-}}^{B} c_{+}=2 \Pi_{+}\left(\nabla_{Y}^{+} Z+g^{-1} c\left(i_{Y} F, t\right)\right)+d_{Y}^{\theta} t-F(Y, Z), \\
& D_{a_{+}}^{B} b_{-}=2 \Pi_{-}\left(\nabla_{X}^{-} Y+g^{-1} c\left(i_{Y} F, r\right)\right), \\
& D_{b_{-}}^{B} d_{-}=2 \Pi_{-}\left(\nabla_{Y}^{-} W\right),
\end{aligned}
$$

where $F$ is the curvature of $\theta$. To calculate the torsion of $D^{B}$, consider the auxiliary covariant derivative

$$
D^{\prime}=\nabla^{g} \oplus d^{\theta} \oplus \nabla^{g^{*}}
$$


MODULI, STROMINGER AND KILLING SPINORS IN GENERALIZED GEOMETRY 37 on $E$, compatible with $V_{+}$. For $e=X+r+\xi$, define

$$
\chi_{e}^{\prime}=-\langle\cdot, \cdot\rangle^{-1} T_{D^{\prime}}=\left(\begin{array}{ccc}
0 & 0 & 0 \\
-i_{X} F & -c^{-1}(c(r,[\cdot, \cdot])) & 0 \\
i_{X} H-2 c(F, r) & 2 c\left(i_{X} F, \cdot\right) & 0
\end{array}\right) \in \mathfrak{o}(E),
$$

(note that $\chi^{\prime}=\chi^{0}$ in the notation of [30]). We also define

$$
\chi_{C}^{\prime} \in E^{*} \otimes \mathfrak{o}(E)
$$

by $\chi_{C e}^{\prime}=\chi_{C(e)}^{\prime}$, which is explicitly given by

$$
\chi_{C e}^{\prime}=\left(\begin{array}{ccc}
0 & 0 & 0 \\
-i_{X} F & 0 & 0 \\
i_{X} H & 2 c\left(i_{X} F, \cdot\right) & 0
\end{array}\right) \in \mathfrak{o}(E) .
$$

Then, we have

$$
D^{B}=D^{\prime}+\left(\chi_{C}^{\prime}\right)^{+++}+\left(\chi^{\prime}\right)^{---}+\left(\chi^{\prime}\right)^{+-+}+\left(\chi^{\prime}\right)^{-+-} .
$$

With the previous formula, a direct calculation using [30, Lemma 3.5] leads us to the following expression for the torsion. Let

$$
\pi_{Q}: E \rightarrow Q=E / T^{*} \cong T P / G
$$

be the natural projection and denote by $C S(\theta) \in \Omega^{3}(P)$ the Chern-Simons 3 -form of $\theta$.

Lemma 5.1. The torsion $T_{D^{B}}$ is the element of $\Lambda^{3} V_{+}^{*} \oplus \Lambda^{3} V_{-}^{*}$ given by

$$
T_{D^{B}}=\pi_{Q \mid V_{+}}^{*}(H-C S(\theta))+\pi_{\mid V_{-}}^{*} H .
$$

More explicitly, taking $a_{+}, c_{+}$as in (5.9) and $b_{+}=Y+s+g Y$, we obtain the formula

$$
\begin{aligned}
T_{D^{B}}^{+}\left(a_{+}, b_{+}, c_{+}\right)= & H(X, Y, Z)+c(r,[s, t]) \\
& -c(F(X, Y), t)+c(F(X, Z), s)-c(F(Y, Z), r),
\end{aligned}
$$

for $T_{D^{B}}=T_{D^{B}}^{+}+T_{D^{B}}^{-}$the natural decomposition. Similarly, setting $a_{-}=$ $X-g X$ we have

$$
T_{D^{B}}^{-}\left(a_{-}, b_{-}, d_{-}\right)=H(X, Y, W) .
$$

We calculate now an explicit formula for the Levi-Civita connection of $V_{+}$. We have

$$
\langle\cdot, \cdot\rangle^{-1} T_{D^{B}}=\left(\chi^{B}\right)^{+++}+\left(\chi^{B}\right)^{---},
$$

where

$$
\chi_{e}^{B}=\left(\begin{array}{ccc}
0 & 0 & 0 \\
-i_{X} F & c^{-1}(c(r,[\cdot, \cdot])) & 0 \\
2 i_{X} H-2 c(F, r) & 2 c\left(i_{X} F, \cdot\right) & 0
\end{array}\right) \in \mathfrak{o}(E) .
$$

Therefore

$$
D^{L C}=D^{B}-\frac{1}{3}\left(\chi^{B}\right)^{+++}-\frac{1}{3}\left(\chi^{B}\right)^{---}
$$


and we conclude that

$$
\begin{aligned}
D_{a_{+}}^{L C} c_{+}= & 2 \Pi_{+}\left(\nabla_{X}^{1 / 3} Z+\frac{2}{3} g^{-1} c\left(i_{X} F, t\right)+\frac{1}{3} g^{-1} c\left(i_{Z} F, r\right)\right) \\
& +d_{X}^{\theta} t-\frac{2}{3} F(X, Z)-\frac{1}{3} c^{-1} c(r,[t, \cdot]), \\
D_{b_{-}}^{L C} c_{+}= & 2 \Pi_{+}\left(\nabla_{Y}^{+} Z+g^{-1} c\left(i_{Y} F, t\right)\right)+d_{Y}^{\theta} t-F(Y, Z), \\
D_{a_{+}}^{L C} b_{-}= & 2 \Pi_{-}\left(\nabla_{X}^{-} Y+g^{-1} c\left(i_{Y} F, r\right)\right), \\
D_{b_{-}}^{L C} d_{-}= & 2 \Pi_{-}\left(\nabla_{Y}^{-1 / 3} W\right) .
\end{aligned}
$$

Remark 5.2. The connection $D^{L C}$ differs by the torsion-free connection $D^{0}$ constructed in [30] by the term $\frac{1}{3}\left(\chi^{\prime \prime}\right)^{+++}$, where

$$
\chi_{e}^{\prime \prime}=\left(\begin{array}{ccc}
0 & 0 & 0 \\
-i_{X} F & 0 & 0 \\
4 c(F, r) & 2 c\left(i_{X} F, \cdot\right) & 0
\end{array}\right) \in \mathfrak{o}(E)
$$

(that vanishes identically in the exact case), as it follows from

$$
\begin{aligned}
D^{L C}= & D^{\prime}+\frac{1}{3}\left(\chi^{\prime}\right)^{+++}+\frac{1}{3}\left(\chi^{\prime \prime}\right)^{+++} \\
& +\frac{1}{3}\left(\chi^{\prime}\right)^{---}+\left(\chi^{\prime}\right)^{+-+}+\left(\chi^{\prime}\right)^{-+-} .
\end{aligned}
$$

This provides a new example of a pair of different torsion-free connections on $E$ compatible with the same metric (cf. [14]). We note that the connection $D^{0}$ is not natural for the action of Aut $E$ (this disproves a claim in [30, Remark 3.8]). For example, for $(B, a) \in$ Aut $E$ we have

$$
\begin{aligned}
\chi^{\prime \prime}\left((B, a) \cdot V_{+}\right)= & \chi^{\prime \prime}\left(V_{+}\right) \\
(B, a)^{-1}\left(\chi_{(B, a) e}^{\prime \prime}\left(V_{+}\right)\right)(B, a) e^{\prime}= & \left(\chi_{e}^{\prime \prime}\left(V_{+}\right)\right) e^{\prime}-2 c(a, F(X, Y)) \\
& +4 c\left(i_{Y} F, a(X)\right)+2 c\left(i_{X} F, a(Y)\right)
\end{aligned}
$$

for $e=X+r+\xi$ and $e^{\prime}=Y+t+\eta$.

Finally, we provide an explicit formula for the connection (5.7). In this work, we are interested in the case that $\varphi$ is an exact 1-form, so we assume $\varphi \in \Omega^{1}(M)$. Then, $D^{\varphi}$ satisfies

$$
\begin{aligned}
& D_{a_{+}}^{\varphi} c_{+}=D_{a_{+}}^{L C} c_{+}+\frac{\left(\operatorname{rk} V_{+}-1\right)^{-1}}{3} \Pi_{+}\left(\varphi(Z) a_{+}-2(g(X, Z)+c(r, t)) \varphi\right), \\
& D_{b_{-}}^{\varphi} c_{+}=D_{b_{-}}^{L C} c_{+}, \\
& D_{a_{+}}^{\varphi} b_{-}=D_{a_{+}}^{L C} b_{-}, \\
& D_{b_{-}}^{\varphi} d_{-}=D_{b_{-}}^{L C} d_{-}+\frac{\left(\operatorname{rk} V_{-}-1\right)^{-1}}{3} \Pi_{-}\left(\varphi(W) b_{-}+2 g(Y, W) \varphi\right) .
\end{aligned}
$$


5.3. The Killing spinor equations. We introduce now the notion of Killing spinor of our interest and study its relation with classical geometry. We will restrict ourselves to the case when $M$ is a six dimensional spin manifold and $E$ is transitive or exact.

As in the previous section, let $E$ be a transitive Courant algebroid, obtained from reduction. Consider an admissible generalized metric $V_{+} \subset E$ such that the corresponding metric on $M$ (see (5.3) ) is positive definite. Then, there exists a positive-definite metric $g$ on $M$ such that

$$
\begin{aligned}
& V_{+}=\{X+g(X)+r: X \in T, r \in \text { ad } P\}, \\
& V_{-}=\{X-g(X): X \in T\},
\end{aligned}
$$

and $\pi_{\mid V_{-}}: V_{-} \rightarrow(T, g)$ induces an anti-isometry. The spin condition $w_{2}(T)=0$ for the manifold $M$ implies the existence of a spinor bundle $S\left(V_{-}\right)$. As $\operatorname{rk} V_{-}$is even, we have a direct sum decomposition into positive and negative chirality half-spinor bundles

$$
S\left(V_{-}\right)=S_{+}\left(V_{-}\right) \oplus S_{-}\left(V_{-}\right) \subset C l\left(V_{-}\right) .
$$

Here, $C l\left(V_{-}\right)$is the Clifford bundle for $V_{-}$, with fibre $C l\left(\left(V_{-\mid x}\right)^{*}\right)$ for any $x \in X$.

Given $\phi \in C^{\infty}(M)$, consider the generalized connection $D^{\phi}=D^{\varphi}\left(V_{+}\right)$determined by $V_{+}$and the 1 -form

$$
\varphi=6 d \phi
$$

By compatibility, $D^{\phi}$ induces differential operators

$$
D_{ \pm}^{\phi}: V_{-} \rightarrow V_{-} \otimes\left(V_{ \pm}\right)^{*}
$$

where we omit, here and below, the symbol of sections for the sake of simplicity. From $D_{+}^{\phi}$ and $D_{-}^{\phi}$ we get differential operators on spinors

$$
D_{ \pm}^{\phi}: S_{+}\left(V_{-}\right) \rightarrow S_{+}\left(V_{-}\right) \otimes\left(V_{ \pm}\right)^{*}
$$

and the associated Dirac operator

$$
\not D_{-}^{\phi}: S_{+}\left(V_{-}\right) \rightarrow S_{-}\left(V_{-}\right) .
$$

Definition 5.3. Given a generalized metric $V_{+}$, as before, and $\phi \in C^{\infty}(M)$, the Killing spinor equations for a spinor $\eta \in S_{+}\left(V_{-}\right)$are given by

$$
\begin{aligned}
& D_{+}^{\phi} \eta=0, \\
& \not D_{-}^{\phi} \eta=0 .
\end{aligned}
$$

Proposition 5.2. The system (5.19) is a natural system of equations in generalized geometry, that is, solutions are exchanged under generalized diffeomorphisms.

Proof. Given a triple $\left(V_{+}, \phi, \eta\right)$ which satisfies (5.19) and $f \in$ Aut $E$, we have to check that the triple $\left(f\left(V_{+}\right), \check{f}_{*} \phi, f_{*} \eta\right)$ is also a solution of the equations. Here, $\check{f}$ is the diffeomorphism on $M$ covered by $f$ (see (4.21)). This follows from the naturality of the Bismut connection $D^{B}$, the torsion $T_{D^{B}}$ and the Weyl term $\chi^{\varphi}$. 
In the next result we provide a more explicit characterization of the Killing spinor equations, that will be applied in Section 5.5 to the Strominger system. Recall that given a generalized metric $V_{+}$, by Proposition 4.5 we obtain a corresponding triple $(g, H, \theta)$.

Lemma 5.2. The Killing spinor equations (5.19) are equivalent to the system

$$
\begin{aligned}
F \cdot \eta & =0 \\
\nabla^{-} \eta & =0, \\
(H+2 d \phi) \cdot \eta & =0, \\
d H-c(F \wedge F) & =0,
\end{aligned}
$$

Proof. We use the anchor map $\pi_{\mid V_{-}}: V_{-} \rightarrow(T, g)$ to identify $S\left(V_{-}\right)$with $S(T)$, so that $F, H$ and $d \phi$, as sections of $\wedge^{\bullet} T^{*} \subset C l(T)=\operatorname{End}(S(T))$, act on the spinor $\eta$.

Let $\left\{e_{1}, \ldots, e_{n}\right\}$ be a local orthonormal frame for $T$. We use the following convention for the Clifford algebra relations

$$
e_{i} e_{j}+e_{j} e_{i}=2 \delta_{i j}
$$

An endomorphism $A \in \operatorname{End}(T)$ satisfies

$$
A=\sum_{i, j=1}^{n} g\left(A e_{i}, e_{j}\right) e^{i} \otimes e_{j},
$$

for $\left\{e^{j}\right\}$ the dual frame of $\left\{e_{j}\right\}$. Since $e^{i} \otimes e_{j}-e^{j} \otimes e_{i} \in \mathfrak{s o}(T)$ embeds as $\frac{1}{2} e^{j} e^{i}$ in the Clifford algebra, an endomorphism $A \in \mathfrak{s o}(T)$ corresponds to

$$
A=\frac{1}{2} \sum_{i<j} g\left(A e_{i}, e_{j}\right) e^{j} e^{i} \in \mathrm{Cl}(T) .
$$

Using (5.13) combined with (5.14) and (5.17), we have

$$
\begin{aligned}
D_{+}^{\phi} \eta & =\nabla^{g} \eta+\frac{1}{2} \sum_{i<j} g\left(\left(\chi^{\prime}\right)^{-+-} e_{i}, e_{j}\right) e^{j} e^{i} \cdot \eta \\
& =\nabla^{g} \eta-\frac{1}{2} \sum_{i<j} H\left(e_{i}, e_{j}, \cdot\right) e^{j} e^{i} \cdot \eta+\sum_{i<j} c\left(F\left(e_{i}, e_{j}\right), \cdot\right) e^{j} e^{i} \cdot \eta \\
& =\nabla^{-} \eta-c(F \cdot \eta, \cdot)
\end{aligned}
$$

and therefore the vanishing of $D_{+}^{\phi} \eta$ is equivalent to the first two equations in (5.20). Similarly,

$$
\begin{aligned}
D_{-}^{\phi} \eta= & \nabla^{-} \eta+\frac{1}{3} \sum_{i<j} H\left(e_{i}, e_{j}, \cdot\right) e^{j} e^{i} \cdot \eta \\
& +\frac{1}{5} \sum_{i<j}\left(d \phi\left(e_{i}\right) e^{j} e^{i} \eta \otimes e^{j}-d \phi\left(e_{j}\right) e^{j} e^{i} \eta \otimes e^{i}\right)
\end{aligned}
$$


and hence, assuming that $D_{+}^{\phi} \eta=0$, we have

$$
\begin{aligned}
\not D_{-}^{\phi} \eta & =\frac{1}{3} \sum_{i<j} H\left(e_{i}, e_{j}, e_{k}\right) e^{k} e^{j} e^{i} \cdot \eta-2 d \phi \cdot \eta \\
& =-(H+2 d \phi) \cdot \eta .
\end{aligned}
$$

The last equation follows from the compatibility condition between $H$ and $\theta$ for any admissible metric (see Proposition 4.5).

5.4. Hull-Strominger's theorem. We study next the consequences of the existence of a Killing spinor (5.19) in terms of complex hermitian geometry. The link is provided by Lemma 5.2 combined with the following result, originally due to Hull and Strominger [39, 63, which constitutes the starting point for the theory of the Strominger system. We give here a different proof, using Gauduchon's formula for the Bismut connection on the canonical bundle [32].

Theorem 5.1 ([63]). Given a real 3-form $H \in \Omega^{3}$ and a real smooth function $\phi \in C^{\infty}(M)$, a solution $(g, \eta)$ of the system

$$
\begin{aligned}
\nabla^{-} \eta & =0, \\
(H+2 d \phi) \cdot \eta & =0,
\end{aligned}
$$

for a non-vanishing half-spinor $\eta \in S_{+}$is equivalent to an $\mathrm{SU}(3)$-structure $(\omega, \Omega)$ on $M$ with integrable almost complex structure $J$, Kähler form $\omega=$ $g(J \cdot, \cdot)$ and holomorphic volume form $\Omega$, satisfying

$$
\begin{aligned}
H & =d^{c} \omega, \\
\phi & =-\frac{1}{2} \log \|\Omega\|_{\omega}-\kappa, \\
d^{*} \omega & =d^{c} \log \|\Omega\|_{\omega},
\end{aligned}
$$

for a suitable real constant $\kappa$.

Proof. We start with a solution $(g, \eta)$ of (15.22), and note that the first equation reduces the holonomy of $\nabla^{-}$to $\mathrm{SU}(3)$. This follows from the equality $\operatorname{Spin}(6)=$ $\mathrm{SU}(4)$ (see also [47, Lem. 9.15] and [47, Rem. 9.12]). Let $(\psi, \omega)$ be the corresponding $\mathrm{SU}(3)$-structure on $M$. Here $\psi$ is a parallel complex 3-form which determines an orthogonal almost complex structure $J$ via (2.1) and $\omega=$ $g(J \cdot, \cdot)$ is the corresponding (parallel) Kähler form.

Using the $\mathrm{SU}(3)$-structure we have an explicit model for the half-spinor bundle $S_{+}$: the Clifford module

$$
S_{+} \cong \Lambda^{0, e v e n}
$$

with Clifford action

$$
\xi \cdot \sigma=\sqrt{2}\left(i_{\left(g^{-1} \xi^{1,0}\right)} \sigma+\xi^{0,1} \wedge \sigma\right)
$$

for $\xi$ a 1-form and $\sigma \in \Lambda^{0, e v e n}$. For SU(3), the space of even parallel spinors is 1-dimensional and $\eta$ is identified with a non-vanishing function ([66]), which we can assume to be 1 . 
Choosing a basis $\left\{d z_{j}, d \bar{z}_{j}\right\}$ of $(1,0)$ and $(0,1)$ forms at a point such that

$$
g=\sum_{j=1}^{3} d z_{j} \otimes d \bar{z}_{j}+d \bar{z}_{j} \otimes d z_{j}
$$

we have

$$
\begin{aligned}
d z_{j} \cdot 1 & =0 \\
d \bar{z}_{j} \cdot 1 & =\sqrt{2} d \bar{z}_{j} \\
d z_{i} \wedge d z_{j} \wedge d \bar{z}_{k} \cdot 1 & =0 \\
d \bar{z}_{i} \wedge d \bar{z}_{j} \wedge d z_{k} \cdot 1 & =\sqrt{2}\left(\delta_{k i} d \bar{z}_{j}-\delta_{k j} d \bar{z}_{i}\right) .
\end{aligned}
$$

This implies

$$
(H+2 d \phi) \cdot 1=2 \sqrt{2}\left(H^{0,3}+\frac{1}{2} \sum_{i<j}\left(H_{\overline{i j i}}^{1,2} d \bar{z}_{j}-H_{\overline{i j j}}^{1,2} d \bar{z}_{i}\right)+\bar{\partial} \phi\right)
$$

where $H=H^{3,0}+H^{2,1}+H^{1,2}+H^{0,3}$ is the decomposition in types with respect to $J$ and

$$
H^{1,2}=\sum_{i<j} H_{\overline{i j} k}^{1,2} d \bar{z}_{i} \wedge d \bar{z}_{j} \wedge d z_{k} .
$$

Then, it follows that the second equation in (5.22) is equivalent to the two conditions

$$
\begin{aligned}
H^{0,3} & =0, \\
i \Lambda_{\omega} H^{1,2} & =-2 \bar{\partial} \phi .
\end{aligned}
$$

Using now that $\nabla^{-}$is unitary and has totally skew-torsion $-H$, by $[32$, Eq. $(2.5 .2)]$,

$$
H=-N+\left(d^{c} \omega\right)^{2,1+1,2},
$$

where $N$ denotes the Nijenhuis tensor of $J$, which is of type $(3,0)+(0,3)[32$, Prop. 1].

Hence, $N=0, H=d^{c} \omega$ and also

$$
\Lambda_{\omega} d \omega=2 d \phi,
$$

where, recall, $\Lambda_{\omega} d \omega=J d^{*} \omega$ is the Lee form of the hermitian structure, and therefore $\omega$ is conformally balanced. Using now Gauduchon's formula [32, Eq. $(2.7 .6)]$

$$
\nabla^{C}=\nabla^{-}+i d^{*} \omega \otimes \mathrm{Id}
$$

relating the connections induced by $\nabla^{-}$and the Chern connection $\nabla^{C}$ on the canonical bundle, combined with $\nabla^{-} \psi=0$, it follows that

$$
\Omega:=e^{-2 \phi} \psi
$$

is a holomorphic volume form for the given complex structure. Finally, from (2.5) we obtain that

$$
\phi=-\frac{1}{2}\left(\log \|\Omega\|_{\omega}-\log \|\psi\|_{\omega}\right)
$$


MODULI, STROMINGER AND KILLING SPINORS IN GENERALIZED GEOMETRY 43

where $\|\psi\|_{\omega}$ is constant and therefore

$$
d^{*} \omega=d^{c} \log \|\Omega\|_{\omega} .
$$

For the converse, we simply define $\eta=1$ in the model for $S_{+}$provided by the $\mathrm{SU}(3)$-structure.

5.5. Metrics with holonomy $\mathrm{SU}(3)$ and the Strominger system. We want to give a complete characterization of (5.19) in terms of complex geometry using Hull-Strominger's theorem, when $E$ is either a exact or a transitive Courant algebroid.

We assume first that $E$ is exact. By definition, $E$ fits into an exact sequence

$$
0 \rightarrow T^{*} \rightarrow E \rightarrow T \rightarrow 0
$$

and the admissible metric $V_{+}$induces a splitting

$$
E=T \oplus T^{*} .
$$

In this splitting, the generalized metric takes the form

$$
\begin{aligned}
& V_{+}=\{X+g(X): X \in T\}, \\
& V_{-}=\{X-g(X): X \in T\},
\end{aligned}
$$

and the induced 3 -form $H$ is closed

$$
d H=0 .
$$

Theorem 5.2. Assume that $E$ is exact. Then $\left(V_{+}, \phi, \eta\right)$ is a solution of (5.19) with $\eta \neq 0$ if and only if $H=0, \phi$ is constant and $g$ is a metric with holonomy contained in $\mathrm{SU}(3)$.

The proof will follow from Hull-Strominger's theorem and Lemma 5.2, which in the present setup specifies to the following result.

Lemma 5.3. Assume that $E$ is exact. Then $(\underline{5.19})$ is equivalent to the system

$$
\begin{aligned}
\nabla^{-} \eta & =0, \\
(H+2 d \phi) \cdot \eta & =0, \\
d H & =0 .
\end{aligned}
$$

Theorem 5.2 is a well-known fact in the literature, that originally appeared in [41, 42, but we sketch here a proof with the complete argument for the convenience of the reader.

Proof of Theorem 5.2. For the 'only if' part, we note that from Theorem 5.1

$$
\Lambda_{\omega} \rho^{C}=-2 \Lambda_{\omega} d d^{c} \phi
$$

where $\rho^{C}$ is the Ricci form of the Chern connection $\nabla^{C}$. On the other hand, (5.25) implies that [2, (2.11)]

$$
\Lambda_{\omega} \rho^{C}=\Lambda_{\omega} \rho^{B}+4 d^{*}\left(J d^{*} \omega\right)+8\left|d^{*} \omega\right|_{\omega}^{2}
$$

where $\rho^{B}$ is the Ricci form of the Bismut connection, and from [56, Th. 1.1] (see also [23, Eq. (18)]) we have

$$
\frac{1}{32} \Lambda^{2}\left(d d^{c} \omega\right)=2 d^{*}\left(J d^{*} \omega\right)+2\left|d^{*} \omega\right|^{2}-\left|d^{c} \omega\right|^{2} .
$$


Using now that $d d^{c} \omega=d H=0$ and that $\nabla^{-}$has holonomy contained in $\mathrm{SU}(3)$ - hence $\rho^{B}=0$-we obtain (cf. [23])

$$
-2 \Lambda_{\omega} d d^{c} \phi=2\left|d^{c} \omega\right|^{2}+4\left|d^{*} \omega\right|^{2} \geqslant 0 \text {. }
$$

Integrating (5.28) over $M$, we conclude that $\omega$ is Kähler, $H=0$ and $\phi$ is constant. Therefore, $g$ is a metric with holonomy contained in $\mathrm{SU}(3)$.

For the converse, we define $\eta=1 \in \Lambda^{0, \text { even }}$ in the model for $S_{+}\left(V_{-}\right)$given by the SU(3)-structure. Then, since $g$ has holonomy SU(3) we have that $\nabla^{-}=\nabla^{g}$ preserves the previous isomorphism and hence we obtain a solution of (5.27) with $H=0$ and $\phi$ constant.

We go now for the transitive case, using the notation introduced in Section 3.1. We make the assumption that the first Pontryagin class of the principal bundle $P$ with respect to the non-degenerate pairing on the Lie algebra of the structure group $\mathfrak{g}=\mathfrak{k} \oplus \mathfrak{g l}(6, \mathbb{R})$

$$
c=2 \alpha^{\prime}\left(-\operatorname{tr}_{\mathfrak{k}}-c_{\mathfrak{g l}}\right)
$$

vanishes $p_{1}(P)=0$ or, equivalently,

$$
p_{1}\left(P_{K}\right)=p_{1}\left(P_{\mathrm{GL}^{+}}\right) .
$$

By [30, Prop. 2.3], this condition determines a canonical exact Courant algebroid

$$
0 \rightarrow T^{*} P \rightarrow \hat{E} \rightarrow T P \rightarrow 0
$$

endowed with a (lifted) $G$-action and non-degenerate pairing $c$ (such that it admits an equivariant isotropic splitting). The transitive Courant algebroid of our interest

$$
0 \rightarrow T^{*} \rightarrow E \rightarrow T \rightarrow 0,
$$

is then obtained from reduction [30, Prop. 2.4] (alternatively, one can apply [13, Th. 1.7] for a direct construction).

On $E$, we consider admissible metrics $V_{+}$such that the metric $g$ on $M$ induced by $V_{+}$is positive definite and the connection $\theta$ on $P$ is a product of a connection $A$ on $P_{K}$ and a $g$-compatible connection $\nabla$ on $P_{\mathrm{GL}^{+}}$. With this ansatz, the compatibility between $\theta$ and $H$ given in Proposition 4.5 reads

$$
d H=2 \alpha^{\prime}\left(-c_{\mathfrak{g l}}(R \wedge R)-\operatorname{tr}_{\mathfrak{k}}\left(F_{A} \wedge F_{A}\right)\right) .
$$

We are ready to prove the main result of this section, which states the equivalence of the Strominger system with the Killing spinor equations.

Proof of Theorem 1.2. Given a solution of (5.19), from Theorem 5.1 we obtain a Calabi-Yau threefold structure $\Omega$ on $M$ and a conformally balanced Kähler form $\omega$ with $H=d^{c} \omega$. Note that by (5.29) $H$ is not closed, and therefore the last part of the argument in the proof of Theorem 5.2 does not apply. Using Lemma 5.2 and the first equation in (5.20), it follows from [66] that

$$
F \wedge \omega^{2}=0, \quad F \wedge \Omega=0
$$

and hence both $A$ and $\nabla$ are hermitian-Yang-Mills connections. Furthermore, since $\nabla g=0$, the inclusion $\mathfrak{s o}(6) \subset \mathfrak{s l}(6, \mathbb{R})$ and (5.29) imply

$$
d d^{c} \omega=2 \alpha^{\prime}\left(\operatorname{tr} R \wedge R-\operatorname{tr}_{\mathfrak{k}} F_{A} \wedge F_{A}\right),
$$


by definition of $c_{\mathfrak{g r}}$. For the converse, given a solution of the Strominger system we define $\theta=A \times \nabla, H=d^{c} \omega$ and $\phi$ by (5.26). Then, the spinor $\eta$ determined by the given $\mathrm{SU}(3)$-structure (see proof of Theorem 5.2) on $M$ satisfies (5.20) and therefore is Killing.

The last part of the statement follows from Proposition 5.2

Theorem 1.2 shows that the Strominger system provides natural equations in generalized geometry. As a direct consequence, we obtain a precise geometric interpretation of the vector spaces $H^{1}\left(\dot{S}^{*}\right)$ and $H^{1}\left(\widehat{S}^{*}\right)$, as spaces of infinitesimal deformations of solutions of the Killing spinor equations (5.1) modulo infinitesimal symmetries of a Courant algebroid.

\section{REFERENCES}

[1] M. C. Abbati, R. Cirelli, A. Manià and P. W. Michor, The Lie group of automorphisms of a principal bundle, J. Geom. Phys. 6 (1989) 215-235.

[2] B. Alexandrov and S. Ivanov, Vanishing theorems on hermitian manifolds, Differential Geom. Appl. 14 (2001) 251-265.

[3] L. B. Anderson, J. Gray and E. Sharpe, Algebroids, heterotic moduli spaces and the Strominger system, JHEP 07 (2014) 37.

[4] B. Andreas and M. Garcia-Fernandez, Solutions of the Strominger system via stable bundles on Calabi-Yau threefolds, Commun. Math. Phys. 315 (2012), no. 1, 153-168.

[5] D. Baraglia, Leibniz algebroids, twistings and exceptional generalized geometry, J. Geom. Phys. 62 (2012) 903-934.

[6] D. Baraglia and P. Hekmati, Transitive Courant Algebroids, String Structures and Tduality, Advances in Theoretical and Mathematical Physics 19 (2015), no. 3, 613-672.

[7] K. Becker and L. Tseng, Heterotic Flux Compactifications and their moduli, Nucl. Phys. B 741 (2006) 162-179.

[8] K. Becker, L. Tseng and S.-T- Yau, Moduli space of torsional manifolds, Nucl. Phys. B 786 (2007) 119-134.

[9] N. Berline, E. Getzler and M. Vergne, Heat Kernels and Dirac Operators, Grundlehren Text Editions, Springer (1992).

[10] H. Bursztyn, G. Cavalcanti and M. Gualtieri, Reduction of Courant algebroids and generalized complex structures, Adv. Math. 211 (2) (2007) 726-765.

[11] E. Calabi, The space of Kähler metrics, Proc. Int. Congr. Math. Amsterdam 2 (1954) 206-207.

[12] P. Candelas, G. Horowitz, A. Strominger, E. Witten, Vacuum Configurations for Superstrings, Nucl. Phys. B 258 (1985) 46-74.

[13] Z. Chen, M. Stienon and P. Xu, On regular Courant algebroids, J. Symp. Geom. 11 (2013) $1-24$.

[14] A. Coimbra, C. Strickland-Constable and D. Waldram, Supergravity as Generalised Geometry I: Type II Theories, JHEP 11 (2011) 91.

[15] A. Coimbra, R. Minasian, H. Triedl and D. Waldram, Generalized geometry for string corrections, JHEP 11 (2014) 160.

[16] M. Cyrier and J. M. Lapan, Towards the massless spectrum of non-Kähler heterotic compactifications, Adv. Theor. Math. Phys. 10 (2007) 853-877.

[17] X. De la Ossa and E. Svanes, Holomorphic bundles and the moduli space of $N=1$ supersymmetric heterotic compactifications, JHEP 10 (2014) 123.

[18] S.K. Donaldson, Anti-self-dual Yang-Mills connections on a complex algebraic surface and stable vector bundles, Proc. London Math. Soc. 50 (1985) 1-26.

[19] A. Douglis and L. Niremberg, Interior estimates for elliptic systems of partial differential equations, Comm. Pure App. Math. (4) 8 (1955) 503-538. 
[20] T. Fei and S.-T. Yau, Invariant Solutions to the Strominger System on Complex Lie Groups and Their Quotients, Commun. Math. Phys. 338 (2015), no. 3, 1183-1195.

[21] M. Fernández, S. Ivanov, L. Ugarte, D. Vassilev, Non-Kähler heterotic string solutions with non-zero fluxes and non-constant dilaton, JHEP 6 (2014) 73.

[22] M. Fernández, S. Ivanov, L. Ugarte, R. Villacampa, Non-Kähler heterotic-string compactifications with non-zero fluxes and constant dilaton, Commun. Math. Phys. 288 (2009) 677-697.

[23] A. Fino and A. Tomassini, On astheno-Kähler metrics, J. London Math. Soc. 83 (2011) 290-308.

[24] D. Freed, Determinants, torsion and strings, Commun. Math. Phys. 107 (1986) 483513.

[25] J.-X. Fu, On non-Kähler Calabi-Yau Threefolds with Balanced Metrics, Proc. Int. Congress of Mathematicians, Hyderabad, India, Volume II, 705-716, Hindustan Book Agency, New Delhi (2010).

[26] J.-X. Fu, J. Li and S.-T. Yau, Balanced metrics on non-Kähler Calabi-Yau threefolds, J. Diff. Geom. 90 (2012) 81-129.

[27] J.-X. Fu and S.-T. Yau, The theory of superstring with flux on non-Kähler manifolds and the complex Monge-Ampère equation, J. Diff. Geom. 78 (2008) 369-428.

[28] J.-X. Fu, L.-S. Tseng and S.-T. Yau, Local heterotic torsional models, Commun. Math. Phys. 289 (2009) 1151-1169.

[29] J.-X. Fu, Z. Wang and D. Wu, Form-Type Calabi-Yau Equations, Math. Res. Lett. 17 (2010), 887-903.

[30] M. Garcia-Fernandez, Generalized connections and heterotic supergravity, Commun. Math. Phys. 332 (2014) 89-115.

[31] M. Garcia-Fernandez, C. Tipler, Deformations of complex structures and the coupled Kähler-Yang-Mills equations, J. Lond. Math. Soc. 89 (2014), no. 3, 779-796.

[32] P. Gauduchon, Hermitian connections and Dirac operators, Bollettino U.M.I. (7) 11-B (1997) 257-288.

[33] R. Goto, Moduli spaces of topological calibrations, Calabi-Yau, hyperKähler, G2 and Spin(7) structures, Int. J. Math. 15 (2004), no. 3, 211-257.

[34] M. B. Green and J. H. Schwarz, Anomaly cancellations in supersymmetric D $=10$ gauge theory and superstring theory, Phys. Lett. B 149 (1984) 117-122.

[35] M. Gualtieri, Generalized Complex Geometry, DPhil thesis, University of Oxford (2004), arXiv:0401221.

[36] _ Branes on Poisson varieties, The many facets of geometry, 368-394, Oxford Univ. Press, Oxford (2010).

[37] _ Generalized Calabi-Yau manifolds, Q. J. Math 54 (2003) 281-308.

[38] L. Huang, On joint moduli spaces. Math. Ann. 302 (1995) 61-79.

[39] C. Hull, Superstring compactifications with torsion and space-time supersymmetry, In Turin 1985 Proceedings "Superunification and Extra Dimensions" (1986) 347-375.

[40] S. Ivanov, Heterotic supersymmetry, anomaly cancellation and equations of motion, Phys. Lett. B 685 (2-3) (2010) 190-196.

[41] S. Ivanov and G. Papadopoulos, Vanishing theorems and string backgrounds, Class. Quant. Grav. 18 (2001) 1089-1110.

[42] A no-go theorem for string warped compactifications, Phys. Lett. B 497 (2001) 309-316.

[43] D. Kaledin and M. Verbitsky, Non-hermitian Yang-Mills connections, Selecta Mathematica 4 (1988) 279-320.

[44] H. J. Kim, Curvatures and holomorphic vector bundles, PhD thesis, University of California, Berkeley (1985).

[45] S. Kobayashi, Differential Geometry of Complex Vector Bundles, Princeton University Press (1987).

[46] S. Kobayashi and K. Nomizu, Foundations of Differential Geometry, Volume I, Interscience Publishers, New York (1963). 
[47] H. Lawson and M. Michelsohn, Spin geometry, 38 of Princeton Mathematical Series, Princeton University Press, Princeton, N. J. (1989).

[48] H. Lee, Strominger's System on non-Kähler hermitian Manifolds, DPhil thesis, University of Oxford (2011).

[49] J. Li and S.-T. Yau, hermitian-Yang-Mills connections on non-Kähler manifolds, Mathematical aspects of string theory (San Diego, Calif., 1986) 560-573, Adv. Ser. Math. Phys. 1, World Sci. Publishing, Singapore (1987).

[50] J. Li and S.-T. Yau, The existence of supersymmetric string theory with torsion, J. Diff. Geom. 70 (2005) 143-181.

[51] R. B. Lockhart and R. C. Mc Owen, On elliptic systems in $\mathbb{R}^{n}$, Acta Mathematica (1) 150 (1983), 125-135.

[52] _ Elliptic differential operators on non-compact manifolds, Annali della Scuola Normale Superiore di Pisa 12 (3) (1985) 409-447.

[53] M. Lübcke and A. Teleman, The Kobayashi-Hitchin correspondence, World Scientific Publishing Co. Inc. (1995).

[54] K. Mackenzie, General theory of Lie groupoids and Lie algebroids, London Mathematical Society Lecture Note Series, no. 213, Cambridge University Press (2005).

[55] D. Martelli and J. Sparks, Non-Kähler heterotic rotations, Adv. Theor. Math. Phys. 15 (1) (2011), 131-174.

[56] K. Matsuo and T. Takahashi, On compact astheno-Kähler manifolds, Colloq. Math. 89 (1) (2001), 213-221.

[57] I. Melnikov and E. Sharpe, On marginal deformations of $(0,2)$ non-linear sigma models, Phys. Lett. B705 (2011) 529-534.

[58] M. L. Michelsohn, On the existence of special metrics in complex geometry, Acta Math. 149 (1) (1982), 261-295.

[59] D. Popovici, Holomorphic Deformations of Balanced Calabi-Yau $\partial \bar{\partial}$-Manifolds, ArXiv preprint, arxiv:1304.0331.

[60] R. Rubio, $B_{n}$-generalized geometry and $G_{2}^{2}$-structures, J. Geom. Phys. 73 (2013) 150156.

[61] Generalized geometry of type $B_{n}$, DPhil thesis, University of Oxford (2014).

[62] H. Sati, U. Schreiber and J. Stasheff, Twisted differential string and fivebrane structures, Commun. Math. Phys. 315 (2012) 169-213.

[63] A. Strominger, Superstrings with torsion, Nucl. Phys. B 274 (2) (1986) 253-284.

[64] L.-S. Tseng and S.-T. Yau, Non-Kähler Calabi-Yau Manifolds, String-Math 2011, 241254, Proc. Symposia in Pure Mathematics 85 (2012).

[65] K. Uhlenbeck and S.-T. Yau, On the existence of hermitian-Yang-Mills connections on stable bundles over compact Kähler manifolds, Comm. Pure and Appl. Math. 39-S (1986) 257-293.

[66] M. Wang, Parallel spinors and parallel forms, Ann. Global Anal. Geom. (1) 7 (1989) 59-68.

[67] F. Witt, Special metric structures and closed forms, DPhil thesis, University of Oxford (2005).

[68] _ Calabi-Yau manifolds with B-fields, Rend. Sem. Mat. Univ. Politec. Torino 66 (2008) 1-21.

[69] S.-T. Yau, Calabi's conjecture and some new results in algebraic geometry, Proc. Natl. Acad. Sci. USA 74 (1977) 1798-1799.

[70] Complex geometry: Its brief history and its future, Science in China Series A Mathematics 48 (2005) 47-60.

[71] _ Metrics on complex manifolds, Sci. China Math. 53 (2010), no. 3, 565-572.

Instituto de Ciencias Matemáticas (CSIC-UAM-UC3M-UCM), Nicolás CabrERA 13-15, Cantoblanco, 28049 Madrid, Spain

E-mail address: mario.garcia@icmat.es 
impa, Estrada Dona Castorina, 110, Jardim Botânico, Rio de Janeiro - RJ, 22460-320, BRAZIL

E-mail address: rubio@impa.br

LMBA, UMR CNRS 6205; DÉPARTEment de Mathématiques, Université de Bretagne Occidentale, 6, Avenue Victor le Gorgeu, 29238 Brest Cedex 3 FRANCE

E-mail address: carl.tipler@univ-brest.fr 